\title{
Stabilized high-order Galerkin methods based on a parameter-free dynamic SGS model for LES
}

\author{
Simone Marras ${ }^{* \dagger} \quad$ Murtazo Nazarov ${ }^{\ddagger} \quad$ Francis X. Giraldo*
}

\begin{abstract}
The high order spectral element approximation of the Euler equations is stabilized via a dynamic sub-grid scale model (Dyn-SGS). This model was originally designed for linear finite elements to solve compressible flows at large Mach numbers. We extend its application to high-order spectral elements to solve the Euler equations of low Mach number stratified flows. The major justification of this work is twofold: stabilization and large eddy simulation are achieved via one scheme only.

Because the diffusion coefficients of the regularization stresses obtained via Dyn-SGS are residual-based, the effect of the artificial diffusion is minimal in the regions where the solution is smooth. The direct consequence is that the nominal convergence rate of the high-order solution of smooth problems is not degraded. To our knowledge, this is the first application in atmospheric modeling of a spectral element model stabilized by an eddy viscosity scheme that, by construction, may fulfill stabilization requirements, can model turbulence via LES, and is completely free of a user-tunable parameter.

From its derivation, it will be immediately clear that Dyn-SGS is independent of the numerical method; it could be implemented in a discontinuous Galerkin, finite volume, or other environments alike. Preliminary discontinuous Galerkin results are reported as well. The straightforward extension to non-linear scalar problems is also described. A suite of 1D, 2D, and $3 \mathrm{D}$ test cases is used to assess the method, with some comparison against the results obtained with the most known Lilly-Smagorinsky SGS model.
\end{abstract}

\section{Introduction}

The search for the best stabilization method for high-order continuous Galerkin (CG) to solve the Euler equations remains an active field of research [24]. This is partially due to certain properties that the stabilization method should have but that are not always present. In particular, if achieved via some type of artificial diffusion, (i) the effect of stabilization on the solution should vanish where the solution is smooth ${ }^{1}$ and (ii) should decrease as the grid is refined. Most stabilized (Galerkin) methods possess the latter but not the first (e.g, [21], spectral elements). To fulfill the first requirement, solution-dependent viscosities have existed for quite some time, especially to stabilize low order finite element [7;29;28;45;27], finite volume [1], and high-order discontinuous

\footnotetext{
*Naval Postgraduate School, Dept. of Applied Mathematics. Monterey (CA) U.S.A.

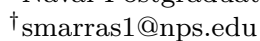

$¥$ Uppsala University, Dept. of Information Technology, Sweden

${ }^{1}$ This property is referred to as consistency.
} 
Galerkin (DG) [44; 34] methods. Residual-based viscosity for spectral elements is found in the extensive work on the entropy-viscosity method by $[22 ; 23]$. Based on the idea of the Spectral Vanishing Viscosity of [50], the entropy-viscosity technique was designed in such a way that (iii) the spectral accuracy of the spatial approximation is preserved if the solution is smooth. This represents the third property that a viscosity-based stabilizing scheme should possess.

A computationally inexpensive sub-grid scale (SGS) model for compressible Large Eddy Simulation (LES) that possesses (i) and (ii) was recently designed to stabilize linear finite elements for different flow regimes [43]. At first sight, this model is a close relative of the entropy-viscosity method, with the fundamental difference that no entropy equation is used to construct the dynamic dissipation. Rather, the residuals of the Euler equations are used to build fully coupled dissipation coefficients. Furthermore, unlike the entropy-viscosity method and, to the best of our knowledge, unlike any of the dissipation models listed above, this scheme converges to the unique entropy solution (see the proof in [42]). The use of the residuals of each equation in the system makes the artificial diffusion non-linear. The reason for exploring non-linear dissipation stems from the analysis reported in [15], where it is concluded that linear stabilization can, at most, give a solution that converges to a weak solution that is not the entropy solution.

In the current paper, we explore the capabilities of this dynamic model (from now on, Dyn-SGS) for the stabilization of the spectral element solution of the Euler equations for low Mach number stratified flows. Furthermore, we extend the method for the solution of quasi-linear scalar equations for use in transport schemes as required in atmospheric modeling.

The derivation of Dyn-SGS is tied to the fundamental idea of LES; the flow equations (Euler, in this particular case) are filtered to separate the large (grid resolved) from the small (sub-grid, unresolved) scales [35]. The filtering operation yields a new set of equations containing additional terms that account for the effect of the unresolved scales onto the resolved solution.

The original version of the current method [43] relies on the regularization of the continuity equation, other than momentum and energy. The idea of mass stabilization is not new; we find it in, e.g., $[44 ; 34 ; 49 ; 5 ; 23 ; 41 ; 24]$. Despite the remarkable results that are shown in the aforementioned literature, this type of regularization is often the subject of criticism by physicists who, for the most part, doubt the physical meaning of mass dissipation on the one hand and the conservation of mass on the other. To not affect conservation, in the current work we build Dyn-SGS so that dissipation is not applied to the continuity equation. Its formal derivation is described in the text. Note that mass may still be conserved when the continuity equation is stabilized, as long as certain boundary conditions are applied.

In Dyn-SGS the diffusion coefficients of the (turbulent) stresses are a function of the equation residuals. In contrast to most artificial diffusion methods that apply the same operator and coefficient to every equation, Dyn-SGS applies separate diffusion coefficients to each equation. Because Dyn-SGS is residual-based, the effect of the artificial diffusion is minimal in the regions where the solution is smooth. The third property (iii) mentioned above is a direct consequence of this.

In spite of, e.g., its higher parallel cost than its second order counterpart and the possible need of a tunable parameter, linear and monolithic ${ }^{2}$ hyper-viscosity remains the classical approach used by atmospheric modelers [31]. A recent review of dissipation-based stabilization schemes for Galerkin methods can be found in [40]. This work represents, to our knowledge, the first application of a non-linear and grid dependent artificial diffusion to stabilize high-order spectral elements in the solution of the Euler equations for stratified flows and that possesses the three properties (i), (ii),

\footnotetext{
${ }^{2}$ Monolithic [24] means that the $2 \alpha$-order operator $(-1)^{\alpha+1} \nabla^{\alpha} \cdot\left(\mu \nabla^{\alpha}\right)$ with diffusion coefficient $\mu$ is applied equally to all quantities.
} 
Table 1: List of constants used in this paper

\begin{tabular}{lcr}
\hline \hline Symbol & Description & Value \\
\hline$c_{p}$ & specific heat of dry air at constant pressure & $1004.5 \mathrm{Jkg}^{-1} \mathrm{~K}^{-1}$ \\
$c_{v}$ & specific heat of dry air at constant volume & $717.5 \mathrm{~J} \mathrm{~kg}^{-1} \mathrm{~K}^{-1}$ \\
$\gamma$ & $c_{p} / c_{v}$ & 1.4 \\
$R$ & gas constant of dry air & $287.17 \mathrm{~J} \mathrm{~kg}^{-1} \mathrm{~K}^{-1}$ \\
$g$ & magnitude of acceleration of gravity & $9.80616 \mathrm{~ms} \mathrm{~s}^{-1}$ \\
$p_{0}$ & reference pressure & $10^{5} \mathrm{~Pa}$ \\
$\omega$ & angular velocity of the Earth & $7.292 \times 10^{-5} \mathrm{~s}^{-1}$ \\
\hline \hline
\end{tabular}

and (iii). Moreover, Dyn-SGS is fully parameter free. The extension to linear and non-linear scalar problems is straightforward and is described as well.

The current method is successfully tested on a series of 1D, 2D and 3D benchmark problems for stratified and gravity driven atmospheres and scalar transport problems.

This study is a first effort towards the construction of an LES model within the Nonhydrostatic Unified Model of the Atmosphere (NUMA) developed by the authors during the past few years $[33 ; 19]$.

The rest of the paper is organized as follows. The sets of equations and the SGS model are described in Section 2. The numerical discretization method of these equations is reported in Section 3. The results for the Euler and scalar equations are reported in Sections 4 and, respectively, 5. The conclusions are given in Section 6 .

\section{Governing equations}

Let $\Omega \in \mathbb{R}^{3}$ be a fixed 3-dimensional domain with boundary $\Gamma$ and Cartesian coordinates $\mathbf{x}=$ $\left[x_{1}(x), x_{2}(y), x_{3}(z)\right]$. Let us identify the dry air density, the velocity vector, and the potential temperature with the symbols $\rho, \mathbf{u}=u_{i}(i=1,2,3)$ and $\theta$. Then, the time-dependent Euler equations in advective form can be written as:

$$
\begin{gathered}
\frac{\partial \rho}{\partial t}+\frac{\partial \rho u_{j}}{\partial x_{j}}=0 \\
\frac{\partial u_{i}}{\partial t}+u_{j} \frac{\partial u_{i}}{\partial x_{j}}+\frac{1}{\rho} \frac{\partial p}{\partial x_{i}}=-g \delta_{i}, \\
\frac{\partial \theta}{\partial t}+u_{j} \frac{\partial \theta}{\partial x_{j}}=0
\end{gathered}
$$

where $g$ is the acceleration due to gravity that acts in the direction of $\delta_{1,2,3}=\left[\begin{array}{ll}0 & 0-1\end{array}\right]$. Equations (1) must be solved in $\Omega \forall t \in \mathbb{R}^{+}$given proper initial and boundary conditions. Pressure, $p$, is related to $\theta$, and $\rho$ through the equation of state for a perfect gas

$$
p=p_{0}\left(\frac{\rho R \theta}{p_{0}}\right)^{\gamma} .
$$

The values of the reference pressure, $p_{0}$, and of the other constants used in the paper are reported in Table 1. 
Remark 1 Because in Numerical Weather Prediction (NWP) the small variations of the thermodynamic quantities $(\rho, \theta, p)$ with respect to a constant and hydrostatically balanced reference background state $\left(\rho_{\text {ref }}, \theta_{\text {ref }}, p_{\text {ref }}\right)$ are the quantities of major interest [38], the splitting $\Delta \rho(t, \mathbf{x})=$ $\rho(t, \mathbf{x})-\rho_{\text {ref }}(z), \Delta \theta(t, \mathbf{x})=\theta(t, \mathbf{x})-\theta_{\text {ref }}(z)$, and $\Delta p(t, \mathbf{x})=p(t, \mathbf{x})-p_{\text {ref }}(z)$ must be introduced to solve Eqs. (1). Nevertheless, to keep the notation clear, the symbol $\Delta$ will only be used in the analysis of the results rather than in the definition and analysis of the LES equations described from now on.

\subsection{LES and the Dyn-SGS model}

The LES formulation is obtained by first introducing the spatial filtering operation

$$
\bar{f}(\mathbf{x})=\int_{\Omega} G_{\bar{\Delta}}(\mathbf{x}-\chi) f(\chi) d \chi,
$$

where the resolved quantity, $\bar{f}$, is obtained from the filtering function $G$ of the instantaneous quantities, $f$, using a filter width $\bar{\Delta}$. The application of (2) to the continuity equation (1a) results in the presence of an additional sub-grid term on the right-hand side. To avoid it, the change of variable $\widetilde{f}=\overline{\rho f} / \bar{\rho}[16]$ is also introduced ${ }^{3}$. The details of the filtering operations for the compressible equations can be found in, e.g. [18] and citations therein. The two operations yield the filtered equations

$$
\begin{gathered}
\frac{\partial \bar{\rho}}{\partial t}+\frac{\partial \bar{\rho} \widetilde{u}_{j}}{\partial x_{j}}=0 \\
\frac{\partial \widetilde{u}_{i}}{\partial t}+\widetilde{u}_{j} \frac{\partial \widetilde{u}_{i}}{\partial x_{j}}+\frac{1}{\bar{\rho}} \frac{\partial \bar{p}}{\partial x_{i}}=-\frac{1}{\bar{\rho}} \frac{\partial \tau_{i j}^{S G S}}{\partial x_{j}}-g \delta_{i}, \\
\frac{\partial \widetilde{\theta}}{\partial t}+\widetilde{u}_{j} \frac{\partial \widetilde{\theta}}{\partial x_{j}}=-\frac{1}{\bar{\rho}} \frac{\partial Q_{j}^{S G S}}{\partial x_{j}},
\end{gathered}
$$

where the derivatives of $\tau_{i j}^{S G S}$ and $Q_{j}^{S G S}$ on the right-hand side of (3b) and (3c) represent the contribution of the unresolved sub-grid scales (SGS). In (3b), $\tau_{i j}^{S G S}$ is the turbulent stress tensor,

$$
\tau_{i j}^{S G S}=\bar{\rho}\left(\widetilde{u_{i} u_{j}}-\widetilde{u_{i}} \widetilde{u_{j}}\right),
$$

which is modeled as a function of the tensor

$$
S_{i j}=\frac{1}{2}\left(\frac{\partial \widetilde{u}_{i}}{\partial x_{j}}+\frac{\partial \widetilde{u}_{j}}{\partial x_{i}}\right),
$$

as

$$
\tau_{i j}^{S G S}=2 \mu S_{i j} .
$$

How the coefficient $\mu$ is constructed defines the SGS method at hand. It will be defined shortly. Similarly, the quantity $Q_{j}^{S G S}$ in (3c) results from filtering Eq. (1c); it is given by

$$
Q_{j}^{S G S}=\bar{\rho}\left(\widetilde{\theta u_{j}}-\widetilde{\theta} \widetilde{u_{j}}\right)
$$

\footnotetext{
${ }^{3}$ From now on, the symbols ${ }^{\top}$ and $\widetilde{\sim}$ will indicate the grid-resolved quantities (large-eddy).
} 
Table 2: Element characteristic length for anisotropic grids

\begin{tabular}{llc}
\hline \hline Formula & Notes & Reference \\
$\overline{\bar{\Delta}}=(\Delta x \Delta y \Delta z)^{1 / 3} / p$ & & {$[13]$} \\
$\bar{\Delta}=f(\Delta x \Delta y \Delta z)^{1 / 3} / p$ & & {$[46]$} \\
where $\quad f=\cosh \left[\frac{4}{27}\left(\ln ^{2} a_{1}+\ln ^{2} a_{2}-\ln a_{1} \ln a_{2}\right)\right]^{1 / 2}$, & & \\
where $\quad a_{1,2}=\Delta_{\text {small } 1,2} / \max (\Delta x, \Delta y, \Delta z) /(N+1)$ & $\Delta_{\text {small1,2 }}$ are the two smallest sizes. & \\
& & $N$ is the order of approximation. See Section 3. \\
\hline \hline
\end{tabular}

and is modeled as

$$
Q_{j}^{S G S}=\kappa \frac{\partial \widetilde{\theta}}{\partial x_{j}},
$$

where the coefficient $\kappa$ will be defined below. To proceed with the construction of $\mu$ and $\kappa$, we introduce the residuals of the filtered equations from which the dissipative operators are excluded. We write:

$$
\begin{gathered}
R(\bar{\rho})=\frac{\partial \bar{\rho}}{\partial t}+\frac{\partial \bar{\rho} \widetilde{u}_{j}}{\partial x_{j}} \\
R\left(\widetilde{u}_{i}\right)=\frac{\partial \widetilde{u}_{i}}{\partial t}+\widetilde{u}_{j} \frac{\partial \widetilde{u}_{i}}{\partial x_{j}}+\frac{1}{\bar{\rho}} \frac{\partial \bar{p}}{\partial x_{i}}+g \delta_{i} \\
R(\widetilde{\theta})=\frac{\partial \tilde{\theta}}{\partial t}+\widetilde{u}_{j} \frac{\partial \widetilde{\theta}}{\partial x_{j}} .
\end{gathered}
$$

The time derivative in (7) can be approximated via a finite difference approximation. As previously done in, e.g., [23] we use a $2^{\text {nd }}$-order backward differentiation formula. Other methods can be used. The coefficients $\mu$ and $\kappa$ are calculated element-wise on every high order element $\Omega_{e}$ given a spectral element approximation of Equations (3) - the spectral element method will be described in Section 3 - In the current model, the filter width is taken as the characteristic size of an element. Simply, given an element $\Omega_{e}$ of order $N$ and edge lengths $\Delta x, \Delta y, \Delta z$ of comparable orders of magnitude, we define the following characteristic length, and hence filter width:

$$
\bar{\Delta}=\min (\Delta x, \Delta y, \Delta z) /(N+1) \text {. }
$$

This definition is sufficient given the scope of the current study; nevertheless, a more proper definition of $\bar{\Delta}$ for LES should be used in future work.

Remark 2: selection of the element characteristic length The choice of the characteristic element size is tied to the aspect ratio of the element at hand. For grids made of relatively regular hexahedra, being $\Delta x, \Delta y$ and $\Delta z$ of the same order of magnitude, the choice is trivial. On the contrary, highly anisotropic grids (usually the case for global circulation models of the Earth atmosphere, or boundary layer grids), stabilization may be greatly affected by an improper choice of $\bar{\Delta}$. Based on our experience, for $\Delta z /(\Delta x, \Delta y) \ll 1$ and $\Delta x / \Delta y \approx 1$, we recommend to split the dynamic diffusion operator into a horizontal and a vertical component. In this way, simulations on anisotropic grids will rely on anisotropic diffusion. Should a splitting operation not be possible, then we recommend the options in Table 2 . 
For the sensible temperature $T=\theta\left(p / p_{0}\right)^{R / c_{p}}$ and one element of characteristic length $\bar{\Delta}$, we start by defining the following quantities:

$$
\left.\mu_{\max }\right|_{\Omega_{e}}=0.5 \bar{\Delta}=\left\||\widetilde{\mathbf{u}}|+\sqrt{\gamma} \frac{\overline{\bar{p}}}{\bar{\rho}}\right\|_{\infty, \Omega_{e}},
$$

where $|\widetilde{\mathbf{u}}|+\sqrt{\gamma \bar{p} / \bar{\rho}}$ is the maximum wave speed and

$$
\left.\mu_{\mathrm{res}}\right|_{\Omega_{e}}=\bar{\Delta}^{2} \max \left(\frac{\|R(\bar{\rho})\|_{\infty, \Omega_{e}}}{\|\bar{\rho}-\widehat{\rho}\|_{\infty, \Omega}}, \frac{\|R(\widetilde{\mathbf{u}})\|_{\infty, \Omega_{e}}}{\|\widetilde{\mathbf{u}}-\widehat{\mathbf{u}}\|_{\infty, \Omega}}, \frac{\|R(\widetilde{\theta})\|_{\infty, \Omega_{e}}}{\|\widetilde{\theta}-\widehat{\theta}\|_{\infty, \Omega}}\right),
$$

where $\widehat{r}$ indicates the space average of the quantity at hand over $\Omega$ and the norms $\|\cdot\|_{\infty, \Omega}$ at the denominator are used for normalization to preserve the correct dimension of the resulting equation. Having $\mu_{\max }$ and $\mu_{\text {res }}$ constructed, we can compute the dynamic coefficients of the viscosity terms as:

$$
\begin{gathered}
\left.\mu\right|_{\Omega_{e}}=\min \left(\mu_{\max }\left|\Omega_{e}, \mu_{\mathrm{res}}\right| \Omega_{e}\right) \\
\left.\kappa\right|_{\Omega_{e}}=\left.\frac{\operatorname{Pr}}{\gamma-1} \mu\right|_{\Omega_{e}}
\end{gathered}
$$

where Pr is an artificial Prandtl number whose value will be defined in Section 4.

The size of the residuals is proportional to $\left(\bar{\Delta}_{\Omega_{e}}\right)^{-1}$ at discontinuities and is relatively small near smooth regions. Moreover, the size of the viscosity terms never exceeds $\mu_{\max }$, which is equivalent to a stable upwind scheme in regions with sharp discontinuities. We briefly illustrate this last point by taking a finite difference approximation of the $1 \mathrm{D}$ advection-diffusion equation with positive velocity $u$ and a diffusion coefficient proportional to $\mu_{\max }$. Given $n$ time steps and a grid of $j$ points, we write the following:

$$
\begin{gathered}
\frac{\partial q}{\partial t}+u \frac{\partial q}{\partial x}-\frac{1}{2} \bar{\Delta} u \frac{\partial^{2} q}{\partial x^{2}} \approx \\
\frac{q_{j}^{n+1}-q_{j}^{n}}{\Delta t}+u \frac{q_{j+1}^{n}-q_{j-1}^{n}}{2 \bar{\Delta}}-\frac{1}{2} u \bar{\Delta} \frac{q_{j+1}^{n}-2 q_{j}^{n}+q_{j-1}^{n}}{\bar{\Delta}^{2}}= \\
\frac{q_{j}^{n+1}-q_{j}^{n}}{\Delta t}+\frac{1}{2} u \frac{q_{j+1}^{n}-q_{j-1}^{n}-q_{j+1}^{n}+2 q_{j}^{n}-q_{j-1}^{n}}{\bar{\Delta}}= \\
\frac{q_{j}^{n+1}-q_{j}^{n}}{\Delta t}+u \frac{q_{j}^{n}-q_{j-1}^{n}}{\bar{\Delta}},
\end{gathered}
$$

where the last expression is an upwinded difference approximation.

Remark 3: a parallel with Lilly-Smagorinsky Going back to Eq. (4) and the definition of $\mu$, the eddy viscosity model due to Lilly and Smagorinsky [37; 47] (valid for low Mach number flows only) reads as follows:

$$
\mu=2 C_{S} \bar{\Delta}^{2}|\mathbf{S}| \sqrt{1-\mathrm{Ri}},
$$


where $C_{S}$ is a constant to be determined (see $[37 ; 36]$ ), $|\mathbf{S}|=\sqrt{2 S_{i j} S_{i j}}$, and Ri is the Richardson number defined as

$$
\operatorname{Ri} \approx \frac{g}{\theta} \frac{\partial \theta}{\partial z} /|\mathbf{S}| \text {. }
$$

Given $\mu, \kappa$ is obtained analogously.

Dyn-SGS and Smagorinsky clearly come from the same root. This means that Dyn-SGS can be implemented with ease within any code that already contains an implementation of Smagorinsky. By theoretical means and physical assumptions, the Smagorinsky constant assumes values $C_{S}=$ $0.2-0.22$; these are twice as large as the values needed for LES calculations of practical importance [9]. The more typical value $C_{S}=0.14$ is used in the Smagorinsky runs reported in Section 4. No user-defined parameter has to be defined for Dyn-SGS.

\subsection{Extension to quasi-linear scalar equations}

The extension to scalar problems is straightforward. We illustrate it for the three-dimensional advection equation of a scalar $q$ :

$$
\frac{\partial q}{\partial t}+u_{j} \frac{\partial q}{\partial x_{j}}=0 \quad j=1,2,3
$$

where $u_{j}$ are the components of a prescribed velocity field. When $q=u_{1}$, and $j=1$, the 1D quasilinear, inviscid Burgers equation ${ }^{4}[4 ; 8]$ is recovered. If we filter (13) as done for the Euler equations, we find the new problem

$$
\frac{\partial \widetilde{q}}{\partial t}+u_{j} \frac{\partial \widetilde{q}}{\partial x_{j}}=-\frac{\partial Q_{j}^{S G S}}{\partial x_{j}} .
$$

As in Eq. (6), $Q_{j}^{S G S}$ is modeled as

$$
Q_{j}^{S G S}=\mu \frac{\partial \widetilde{q}}{\partial x_{j}}
$$

where $\mu$ is the residual-dependent function

$$
\left.\mu\right|_{\Omega_{e}}=0.5 \bar{\Delta}^{2} \frac{\|R(\widetilde{q})\|_{\infty, \Omega_{e}}}{\|\widetilde{q}-\widehat{q}\|_{\infty, \Omega}} .
$$

\section{Variational formulation and spectral element approxima- tion}

Equations (3) are solved via the spectral element method in the domain $\Omega$ with boundary $\Gamma$. To proceed, we define the following notation: given the Sobolev space $H^{1}(\Omega)$ of weakly-differentiable functions, the space $\mathcal{V} \subseteq H^{1}$ of test and trial functions of the Galerkin formulation is introduced. Given the space $L^{2}$ of real-valued functions that are square integrable in $\Omega$, the 2-norm associated with it is denoted by $\|\cdot\|_{2}$. Given a finite element partition $\Omega^{h}=\cup_{e=1}^{n_{e}} \Omega_{e}$ of the domain $\Omega$ into $n_{e}$ conforming hexahedra $\Omega_{e}$ of characteristic length $\bar{\Delta}, \mathcal{V}^{h}$ is the finite dimensional projection of $\mathcal{V}$.

\footnotetext{
${ }^{4}$ Although it is classically referred to as the "Burgers" equation, the first time that it was introduced dates back to the 1915 work by Bateman [4].
} 
To proceed, let us recast Eqs. (3) in compact form as

$$
\frac{\partial \overline{\mathbf{q}}}{\partial t}+\mathcal{L}(\overline{\mathbf{q}})=-\mathbf{g}
$$

where $\overline{\mathbf{q}}=\left[\bar{\rho}, \widetilde{u}_{i}, \widetilde{\theta}\right]^{T}$ is the array of the solution variables $(i=1,2,3)$ and $\mathcal{L}(\mathbf{q})$ contains all the differential operators that are easily identifiable in the system above. From now on, the symbols

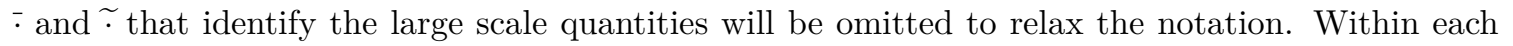
element, the finite dimensional approximation of $\mathbf{q}(\mathbf{x}, t)$ is given by the expansion

$$
\left.\mathbf{q}^{h}(\mathbf{x}, t)\right|_{\Omega_{e}}=\left.\sum_{k=1}^{(N+1)^{3}} \psi_{k}(\mathbf{x}) \mathbf{q}_{k}(t)\right|_{\Omega_{e}}
$$

where $(N+1)^{3}$ is the number of collocation points within the element of order $N$ and $\psi_{k}$ are the interpolation polynomials evaluated at point $k$. The basis functions $\psi_{k}$ are constructed as the tensor product of the one-dimensional functions $h_{\alpha}(\xi(\mathbf{x})), h_{\beta}(\eta(\mathbf{x}))$, and $h_{\gamma}(\zeta(\mathbf{x}))$ as:

$$
\psi_{k}=h_{\alpha}(\xi(\mathbf{x})) \otimes h_{\beta}(\eta(\mathbf{x})) \otimes h_{\gamma}(\zeta(\mathbf{x})), \quad \forall \alpha, \beta, \gamma=0, \ldots, N .
$$

The functions $h_{\alpha}(\xi(\mathbf{x})), h_{\beta}(\eta(\mathbf{x})), h_{\gamma}(\zeta(\mathbf{x}))$ are the basis functions associated with the $N+1$ Legendre-Gauss-Lobatto (LGL) points $\xi_{\alpha}, \eta_{\beta}, \zeta_{\gamma}$, respectively, which are given by the roots of

$$
\left(1-\boldsymbol{\xi}^{2}\right) P_{N}^{\prime}(\boldsymbol{\xi})=0
$$

where $P_{N}^{\prime}(\boldsymbol{\xi})$ are the derivatives of the $N^{t h}$-order Legendre polynomial [14;32]. Given these definitions, the one-dimensional Lagrange polynomials $h_{\alpha, \beta, \gamma}(\boldsymbol{\xi})$ are

$$
h_{\alpha, \beta, \gamma}(\boldsymbol{\xi})=-\frac{1}{N(N+1)} \frac{\left(1-\boldsymbol{\xi}^{2}\right) P_{N}^{\prime}(\boldsymbol{\xi})}{\left(\boldsymbol{\xi}-\boldsymbol{\xi}_{\alpha, \beta, \gamma}\right) P_{N}\left(\boldsymbol{\xi}_{\alpha, \beta, \gamma}\right)} .
$$

The same expansion (17) is used to construct the derivatives. By differentiation of Eq. (17) with respect to time and space, we write:

$$
\begin{aligned}
\left.\frac{\partial \mathbf{q}^{h}(\mathbf{x}, t)}{\partial t}\right|_{\Omega_{e}} & =\sum_{k=1}^{(N+1)^{3}} \psi_{k}(\mathbf{x}) \frac{d \mathbf{q}_{k}(t)}{d t} \\
\left.\frac{\partial \mathbf{q}^{h}(\mathbf{x}, t)}{\partial \mathbf{x}}\right|_{\Omega_{e}} & =\sum_{k=1}^{(N+1)^{3}} \frac{\partial \psi_{k}(\mathbf{x})}{\partial \mathbf{x}} \mathbf{q}_{k}(t) .
\end{aligned}
$$

The definitions and expansions introduced above yield the weak, element problem

$$
\int_{\Omega_{e}^{h}} \psi^{h}\left[\frac{\partial \mathbf{q}^{h}}{\partial t}+\mathcal{L}\left(\mathbf{q}^{h}\right)\right] d \Omega_{e}^{h}=\int_{\Omega_{e}^{h}} \psi^{h} \mathbf{g} d \Omega_{e}^{h} .
$$

By virtue of the global assembly procedure, the global problem is solved on $\Omega^{h}$ and consists in finding $\mathbf{q}^{h}(t, \mathbf{x}) \in \mathcal{V}^{h}$ such that

$$
\int_{\Omega^{h}} \psi^{h}\left[\frac{\partial \mathbf{q}^{h}}{\partial t}+\mathcal{L}\left(\mathbf{q}^{h}\right)\right] d \Omega^{h}=\int_{\Omega^{h}} \psi^{h} \mathbf{g} d \Omega^{h} \quad \forall \psi^{h} \in \mathcal{V}^{h} .
$$


Notice that we have not explicitly treated the second order operators so far. Let us start with the momentum equation and treat the strain

$$
\frac{1}{\rho} \frac{\partial \tau_{i j}^{S G S}}{\partial x_{j}}=\frac{\mu}{\rho} \frac{\partial}{\partial x_{j}}\left(\frac{\partial u_{i}}{\partial x_{j}}+\frac{\partial u_{j}}{\partial x_{i}}\right)
$$

where the dissipative coefficient $\mu$ is taken as a local constant (at the current time step) that can hence be taken out of the derivation. Multiplication by the test function $\psi^{h}$ and integration yields

$$
\int_{\Omega_{e}} \psi^{h} \frac{\mu}{\rho} \frac{\partial}{\partial x_{j}}\left(\frac{\partial u_{i}}{\partial x_{j}}+\frac{\partial u_{j}}{\partial x_{i}}\right) d \Omega_{e},
$$

that becomes, after integration by parts,

$$
\int_{\Gamma_{e}} \psi^{h} \frac{\mu}{\rho} n_{j, \Gamma_{e}}\left(\frac{\partial u_{i}}{\partial x_{j}}+\frac{\partial u_{j}}{\partial x_{i}}\right) d \Gamma_{e}-\int_{\Omega_{e}} \mu \frac{\partial\left(\psi^{h} / \rho\right)}{\partial x_{j}}\left(\frac{\partial u_{i}}{\partial x_{j}}+\frac{\partial u_{j}}{\partial x_{i}}\right) d \Omega_{e},
$$

where $n_{j, \Gamma_{e}}$ is the $j$ component of the unit normal vector on the element boundary and

$$
\frac{\partial\left(\psi^{h} / \rho\right)}{\partial x_{j}}=\frac{1}{\rho} \frac{\partial \psi^{h}}{\partial x_{j}}+\psi^{h} \frac{\partial(1 / \rho)}{\partial x_{j}} .
$$

Because the stresses in the current method represent an artificial diffusion that is only an approximation to the actual Navier-Stokes stresses, to limit the operation count in our implementation we omit the second term on the right hand of side of (22). Furthermore, because of the continuity of the solution across the element boundaries and because of the inviscid or periodic boundary conditions that we will apply in the tests below, the flux term that arises from the integration by parts vanishes.

The final semi-discrete matrix system results from the global integral equation (20) using the expansions (18) and the global assembly of the element matrices that result from it. The system written in compact matrix-vector form is

$$
\frac{d \mathbf{q}_{k}}{d t}=\mathbf{M}^{-1} \mathcal{L}_{k}\left(\mathbf{q}_{k}\right)
$$

where $\mathbf{M}$ is the global mass matrix that is diagonal by construction since the integration and interpolation points within the elements are co-located. For more details on the construction of this system and of the global assembly operation see, e.g., [33].

System (23) is discretized in time by the implicit-explicit (IMEX) time integration described in [19]. Leaving the details of IMEX to [19], the IMEX time approximation uses an implicit

approximation of the (linear) terms responsible for the fast moving acoustic and gravity waves, whereas the slow non-linear advection is treated explicitly. In this paper, IMEX is based on a fourth order multi-stage additive Runge-Kutta (ARK4) method. This approach allows us to relax the constraint that fast waves put on the Courant-Friedrichs-Lewy [12] condition and on the size of the time-step.

\section{Model verification: Euler equations}

In the following section, Dyn-SGS is tested against a suite of standard benchmarks of ubiquitous use when testing new atmospheric dynamical cores. 
First, we perturb a neutrally stable atmosphere with thermal anomalies that vary in definition and size. The tests are divided into $2 \frac{1}{2} \mathrm{D}$, where the domain extends to infinity along the $y$-direction, and fully $3 \mathrm{D}$ problems in a simply connected domain. They include the following: (i) $2 \frac{1}{2} \mathrm{D}$ rising thermal bubble in a large domain (see the original 2D test in [2]) and (ii) the classical density current [48]. In 3D we have: (iii) a rising thermal bubble in a small domain [33], (iv) the baroclinic wave in a channel [51]. Except for the balanced initial state of the baroclinic wave in a channel, an analytic solution does not exist for these problems. For this reason, it must be understood that most of these tests can only give a qualitative (and relative) information on the accuracy that one model can achieve.

\section{1 $2 \frac{1}{2} \mathrm{D}$ rising thermal bubble in a large domain}

This test consists of a flow that is triggered by the thermal perturbation of a neutrally stratified atmosphere at initially uniform potential temperature $\theta_{0}=300 \mathrm{~K}$ and in hydrostatic equilibrium such that the pressure decreases with $z$ as:

$$
p=p_{0}\left(1-\frac{g}{c_{p} \theta_{0}} z\right)^{c_{p} / R}
$$

The domain $\Omega=[-5000,5000] \times[-\infty, \infty] \times[0,10000] \mathrm{m}^{3}$ and the definition of the perturbation are given as in [2]. The perturbation is linear and defined as

$$
\Delta \theta=\theta_{c}\left[1.0-\frac{r}{r_{0}}\right] \quad \text { if } r \leq r_{0}=2000 \mathrm{~m},
$$

where $r=\sqrt{\left(x-x_{c}\right)^{2}+\left(z-z_{c}\right)^{2}},\left(x_{c}, z_{c}\right)=(0,2000) \mathrm{m}$, and $\theta_{c}=2 \mathrm{~K}$. Due to the symmetry of the problem, we simulate only half of the domain (to verify that the current method can indeed preserve symmetry, in Section 4.3 we analyze a fully 3D simulation without any geometric assumptions). The initial velocity field is zero everywhere. Periodic boundary conditions are used along $y$ whereas no-flux is imposed in $x$ and $z$. We perform four runs on four different grids with effective resolutions 1) $\Delta x=\Delta z=125 \mathrm{~m}$ (as in [2]), 2) $\Delta x=\Delta z=62.5 \mathrm{~m}, 3) \Delta x=\Delta z=31.25 \mathrm{~m}$, and 4) $\Delta x=\Delta z=$ $15.625 \mathrm{~m}$. The different resolutions were used to analyze the behavior of the method as the grid is refined, although no proper convergence study is made. The contour lines of the perturbation potential temperature $\Delta \theta$ are plotted at $t=1020 \mathrm{~s}$ in Fig. 1 . The maximum of $\Delta \theta$ at the final time is $\approx 1.4 \mathrm{~K}$, which agrees with the $f$-wave solution of [2] at the same resolution of $125 \mathrm{~m}$, and is equivalent with the WRF simulation reported in the same paper. The extrema of $\Delta \theta$ in this study increase a few fractions of a degree as the grid is refined to $15.625 \mathrm{~m}$. This is to be attributed to the grid dependence of the current SGS model, whose dissipation properties vary quadratically with respect to the size of the grid. We will touch more on this issue in the analysis of the density current below. To maintain the WRF solution stable, Ahmad and Lindeman had to add a constant $15 \mathrm{~m}^{2} \mathrm{~s}^{-1}$ diffusion. They did not for their finite volume-based $f$-wave decomposition. Without a constant diffusion, we preserve stability for all the tested resolutions. The shear between the boundary of the perturbation and the background at rest triggers very well defined Rayleigh-Taylor instabilities that become ever more visible when the resolution is increased. To give some hints to the reader on what the sub grid scales look like and what spatial distribution they have with respect to the developing bubble, we show them in Fig. 2. In the figure, the symbol $S G S(\cdot)$ indicates the 
stresses that appear in the momentum and $\theta$ equations. They are (with some abuse of notation)

$$
S G S\left(u_{i}\right)=\frac{\partial \tau_{i j}^{S G S}}{\partial x_{j}} /\left\|S G S\left(\widetilde{u}_{i}\right)\right\|_{\infty, \Omega}, \quad \text { and } \quad S G S(\theta)=\frac{\partial Q_{j}}{\partial x_{j}} /\|S G S(\widetilde{\theta})\|_{\infty, \Omega} .
$$

In [43] the Prandtl number is assigned the artificial value 0.1. Because this value is fully artificial and does not have a physical foundation, for this study we decided to test how the current stresses behave if we picked the air Prandtl number, approximately equal to 0.7. We also tried 0.1 and an intermediate value of 0.35 . With $\mathrm{Pr}=0.1$ the solution still preserves stability for all the flow regimes that we tested, confirming that the method was implemented properly since 0.1 was successfully used in [43]. However, in our opinion the solution lost some of its physics since hardly no dissipation of $\Delta \theta$ could be observed as time evolved. In other words, the maximum of $\Delta \theta$ was preserved along the full simulation, which is an unphysical behavior for the flows we are interested in simulating. If we looked at this from a mathematical point of view, this is arguably a great result since the method achieved stability without really affecting the originally inviscid system; however, from a more physical point of view, we want a method that not only stabilizes, but also introduces the necessary sub-grid stresses that model the physical dissipation of a moving atmosphere in a sheared environment. In Fig. 3 we compare the solution of the same rising thermal bubble already shown in Fig. 1 for $\mathrm{Pr}=0.35$, but now also using $\mathrm{Pr}=0.7$ (top row of Fig. 3). As expected, $\mathrm{Pr}=0.7$ gave a smoother solution, possibly indicating a greater dissipation. However, excluding the extremely small oscillation that are visible in certain regions in the case of $\operatorname{Pr}=0.35$, we observe how the distributions of $\Delta \theta$ for the two cases are practically identical. The extrema of $\Delta \theta$ are preserved; this is seen by looking at the differences: $\left[\Delta \theta_{\max , \operatorname{Pr}=0.35}-\Delta \theta_{\max , \operatorname{Pr}=0.7}\right](15.625 \mathrm{~m})=0.028 \mathrm{~K}$ and $\left[\Delta \theta_{\text {max }, P r=0.35}-\Delta \theta_{\max , P r=0.7}\right](31.25 \mathrm{~m})=0.1 \mathrm{~K}$; they possibly show that the more physical $\operatorname{Pr}$ and the dynamically adapting diffusion coefficients have an effect on the smaller scales (i.e. small oscillation in the domain) rather than on the global solution and indeed help smoothen the solution only where oscillations are important.

\section{$4.2 \quad 2 \frac{1}{2} \mathrm{D}$ Density current}

The density current was introduced in [10] and became a standard benchmark in the development of atmospheric codes [48]. Like in [2], in this paper the benchmark is run without the constant and uniform artificial diffusion with coefficient $\mu=75 \mathrm{~m}^{2} \mathrm{~s}^{-1}$ of [48]. This is because we are interested in assessing Dyn-SGS as a stabilizing tool that does not require additional constant viscosity. For this reason, no converged solution should be expected. On the contrary, as the grid is refined, more structures will be resolved. We will show this shortly. The background initial state is at a uniform potential temperature $\theta_{0}=300 \mathrm{~K}$ within the domain $\Omega=[-25600,25600] \times[-\infty, \infty] \times[0,6400] \mathrm{m}^{3}$. A perturbation of $\theta$ centered in $\left(x_{c}, z_{c}\right)=(0,3000) \mathrm{m}$ and with radii $\left(r_{x}, r_{z}\right)=(4000,2000) \mathrm{m}$ is given by the function

$$
\Delta \theta=\frac{\theta_{c}}{2}\left[1+\cos \left(\pi_{c} r\right)\right] \quad \text { if } r \leq 1
$$

where $\theta_{c}=-15 \mathrm{~K}$ and $r=\sqrt{\left(x-x_{c}\right) / r_{x}^{2}+\left(z-z_{c}\right) / r_{z}^{2}}$. Periodic boundary conditions are used along $y$ whereas no-flux and free-slip conditions are set in $x$ and $z$. The initial velocity is zero. The time evolution of the density current for $5400 \mathrm{~s}$ is shown in Fig. 4. Because $\Delta \theta$ is transported (and diffused) by the flow, it is the perfect variable to be used in the visualization of mixing. The evolution and transition onto a fully mixed flow is evident. The mixing is triggered by the shear 

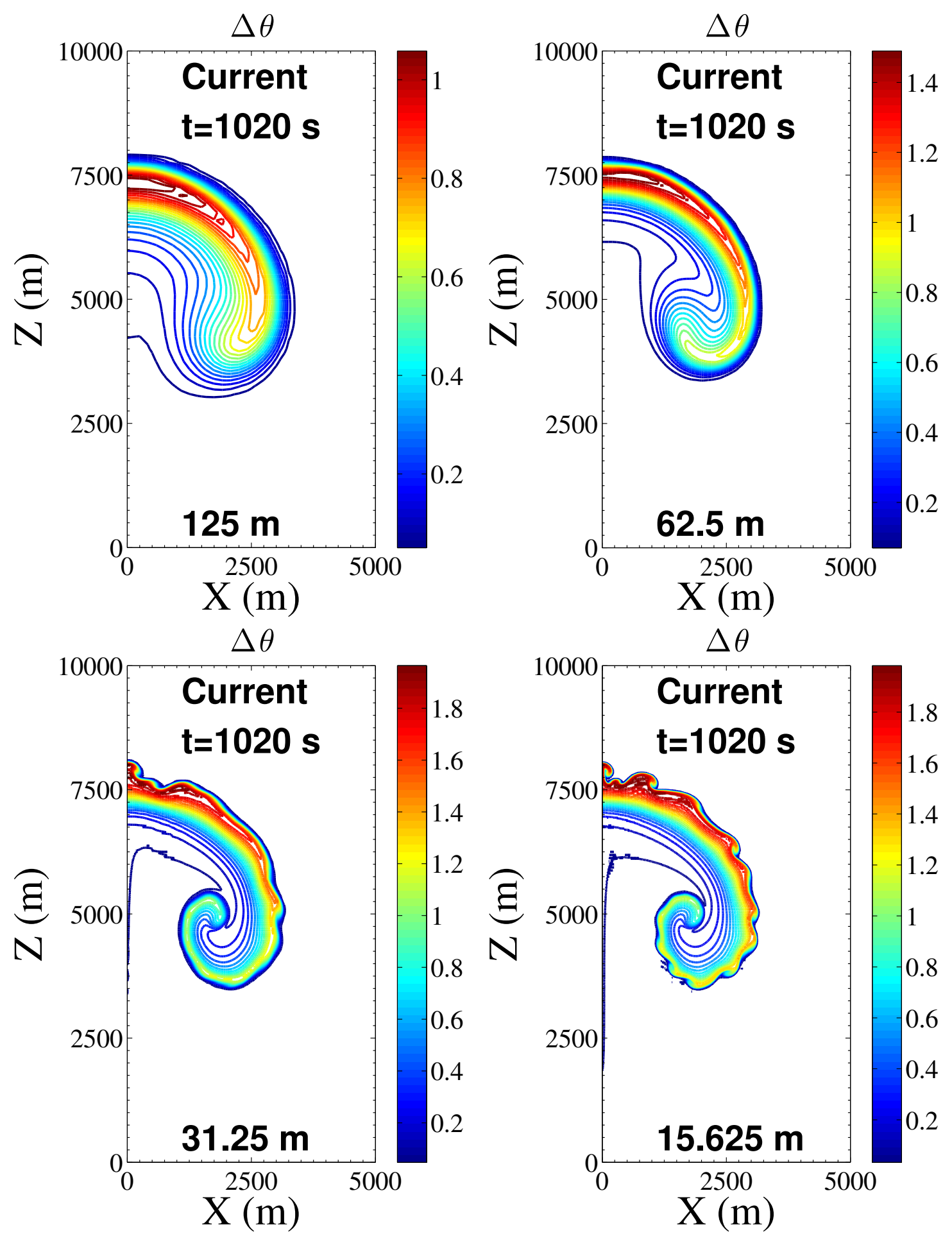

Figure 1: $2 \frac{1}{2} \mathrm{D}$ rising thermal bubble in a large domain. Four resolutions as indicated in each subfigure. The perturbation of potential temperature $\Delta \theta$ is plotted at $t=1020 \mathrm{~s}$. All the results are shown for a maximum Courant number 8. 

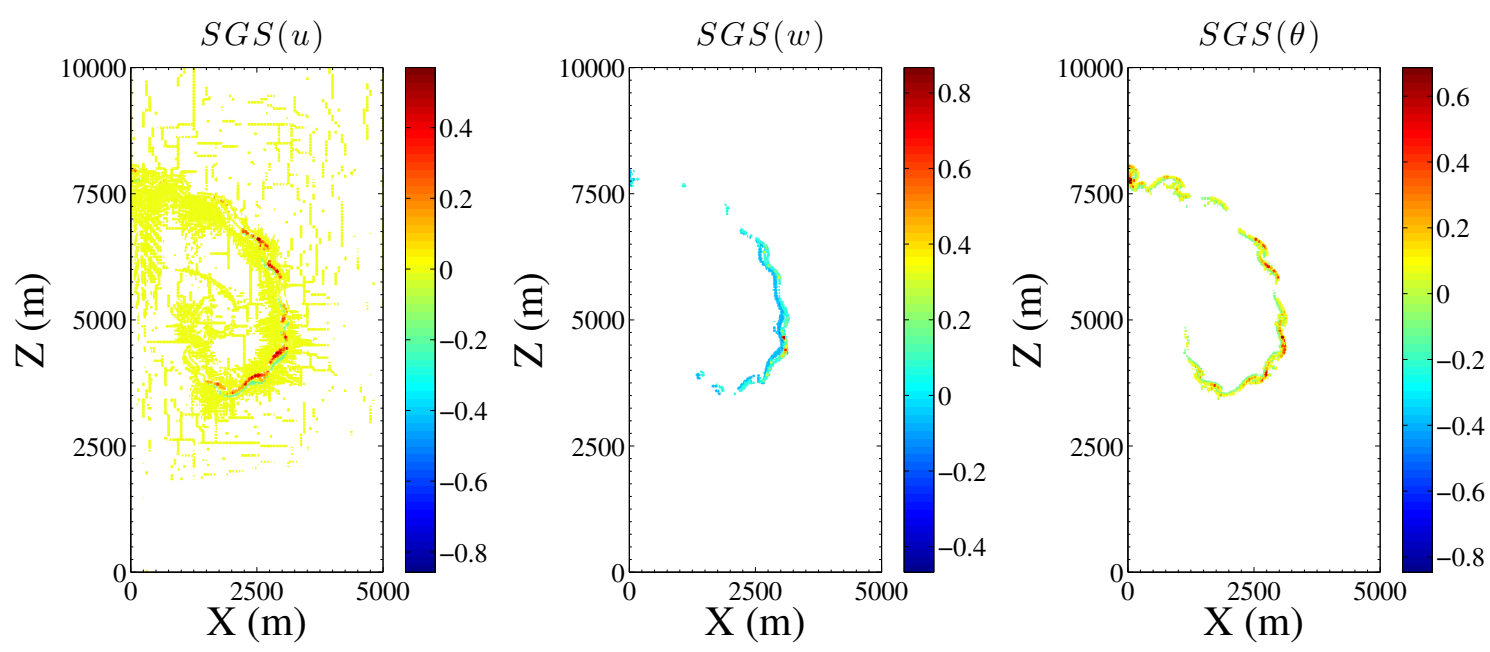

Figure 2: $2 \frac{1}{2} \mathrm{D}$ rising thermal bubble in a large domain. From left to right: contours of the dynamic stabilization stresses $S G S(u), S G S(w)$, and $S G S(\theta)$. The values of the SGS terms are normalized with respect to the global maximum of the stress at hand (i.e. $S G S(\theta)$ is the stress of the $\widetilde{\theta}$ equation and is normalized with respect to its maximum value at the given time step). These plots show the structure of these stresses and how they dynamically adapt to the solution. These stresses are obtained from the fine grid simulation using the resolution $\Delta x=\Delta z=15.625 \mathrm{~m}$.

that becomes greater as the velocity of the moving cold mass of air first accelerates and later hits the right wall. It bounces off the boundary and keeps moving left- and rightwards as more mixing occurs at the expense of kinetic energy. Due to mixing and dissipation, the minimum of $\Delta \theta$ increases from $-15 \mathrm{~K}$ at $t=0 \mathrm{~s}$ to $-3.729 \mathrm{~K}$ during 5400 seconds (from dark blue to light green shading in the plots), whereas the maximum is preserved to $0 \mathrm{~K}$ (dark red).

Figure 5 shows $\Delta \theta$ for the effective resolutions $\Delta x=\Delta z=50 \mathrm{~m}, \Delta x=\Delta z=25 \mathrm{~m}$, and $\Delta x=$ $\Delta z=12.5 \mathrm{~m}$ at simulation times 600,750 , and $900 \mathrm{~s}$. To our knowledge, no data is available to compare against at the $12.5 \mathrm{~m}$ resolution. It is evident that the amount of vortical structures is larger at higher resolution. Certainly, the finer resolution is expected to yield a more resolved solution. However, we believe this to be a mixed effect of the resolution and smaller dissipation required. Just like the classical Smagorinsky and other models, the current SGS model is a quadratic function of the grid size so that dissipation decreases as the grid is refined. The effect on the solution is non-linear. The non-linearity can be explained as follows: the finer grid lowers the influence of dissipation with a direct effect on the oscillations of the solution variable. Slightly larger, yet controlled, localized oscillations imply larger gradients (and hence residuals) that directly affect the dissipation in the regions of larger residuals.

Given the accepted use of the Smagorinsky model $[37 ; 47]$ in numerical weather prediction, to support our hypothesis that a properly designed SGS model can serve as a stabilization method, we compare the results of the current model with the constant coefficient Smagorinsky at $25 \mathrm{~m}$ and $50 \mathrm{~m}$ resolutions. The results are plotted in Figs. 6-7. The two methods are in strong agreement at both resolutions. It must be kept in mind that the density current described here is not a boundary layer flow; the boundaries are treated as if the problem were inviscid although the flow 

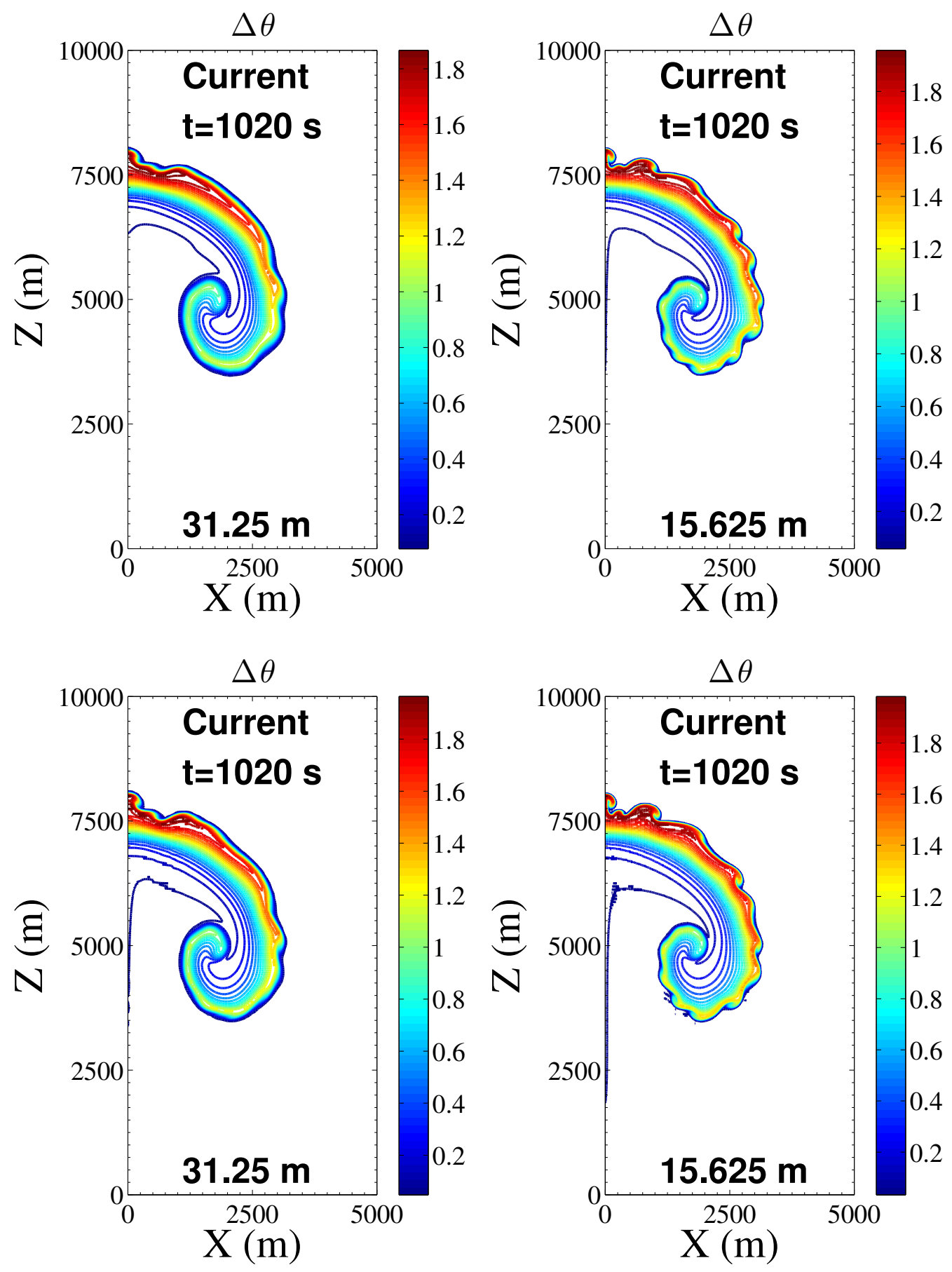

Figure 3: As Fig. 1, but here we concentrate on the effect of the value of the Prandtl number. Top row: $\operatorname{Pr}=0.7$. Bottom row: $\operatorname{Pr}=0.37$. Left column: $\Delta x=\Delta z=31.25$ m. Right column: $\Delta x=\Delta z=$ $15.625 \mathrm{~m}$. As expected the $\operatorname{Pr}=0.7$ solution is smoother, possibly indicating a greater dissipation. However the differences $\left[\Delta \theta_{\max , \operatorname{Pr}=0.35}-\Delta \theta_{\max , \operatorname{Pr}=0.7}\right](15.625 \mathrm{~m})=0.028 \mathrm{~K}$ and $\left[\Delta \theta_{\max , \operatorname{Pr}=0.35}-\right.$ $\left.\Delta \theta_{\max , \operatorname{Pr}=0.7}\right](31.25 \mathrm{~m})=0.1 \mathrm{~K}$ show that the greater Pr has an effect on the smaller scales only rather than on the global solution. 


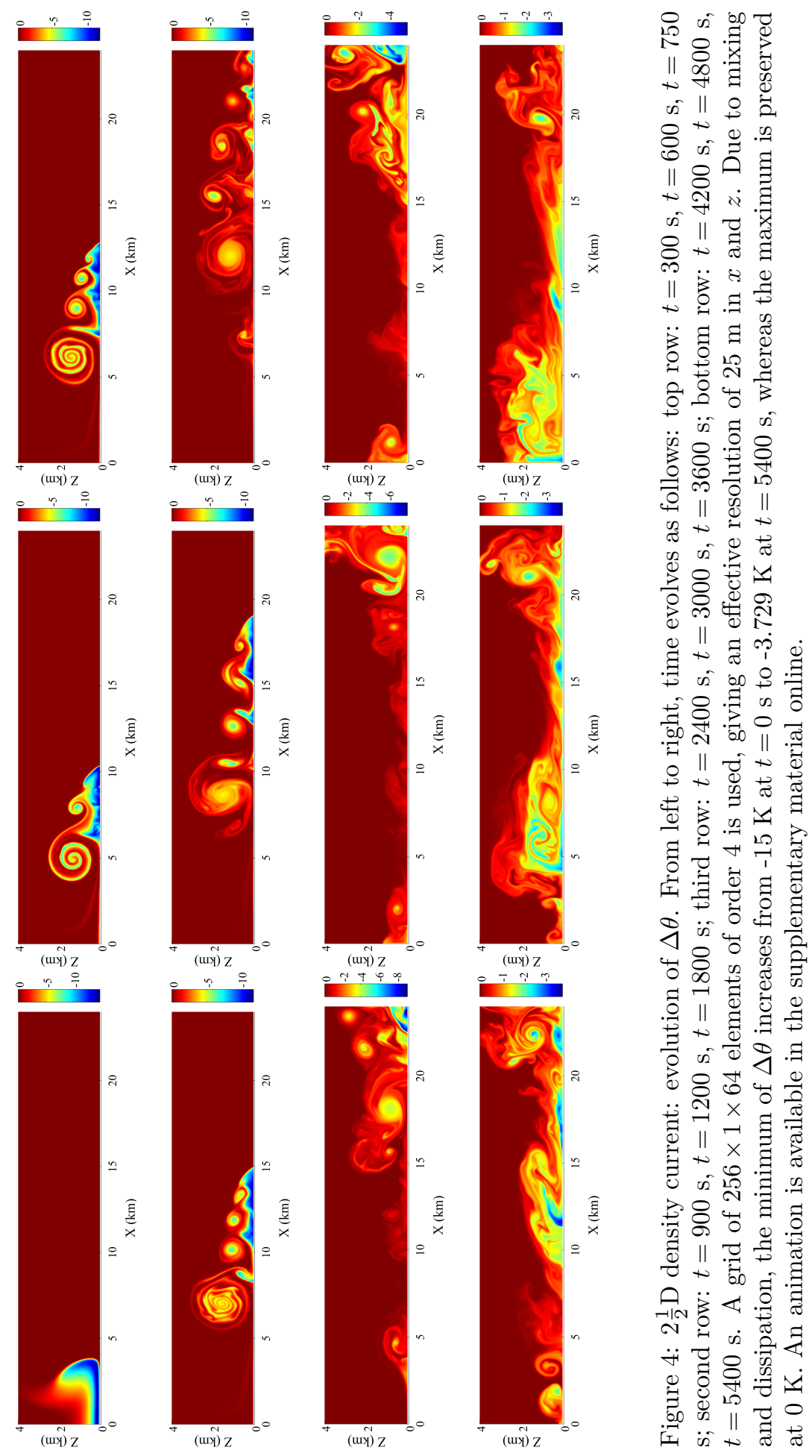




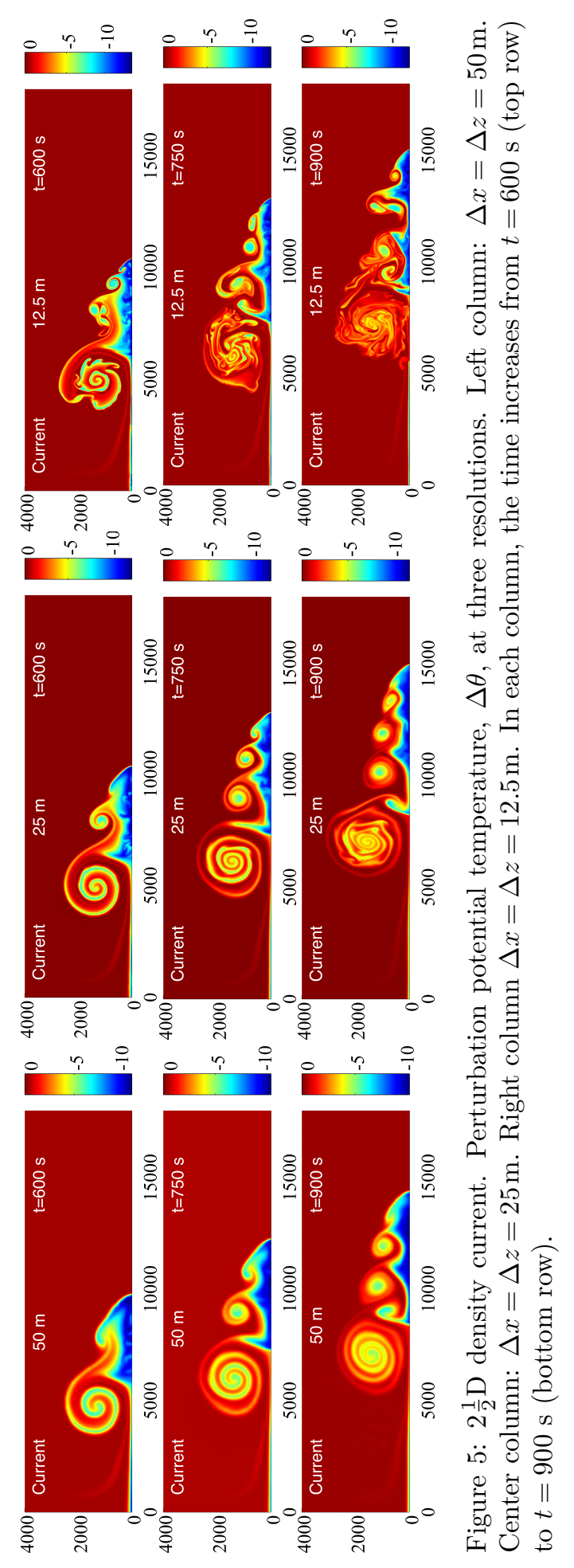




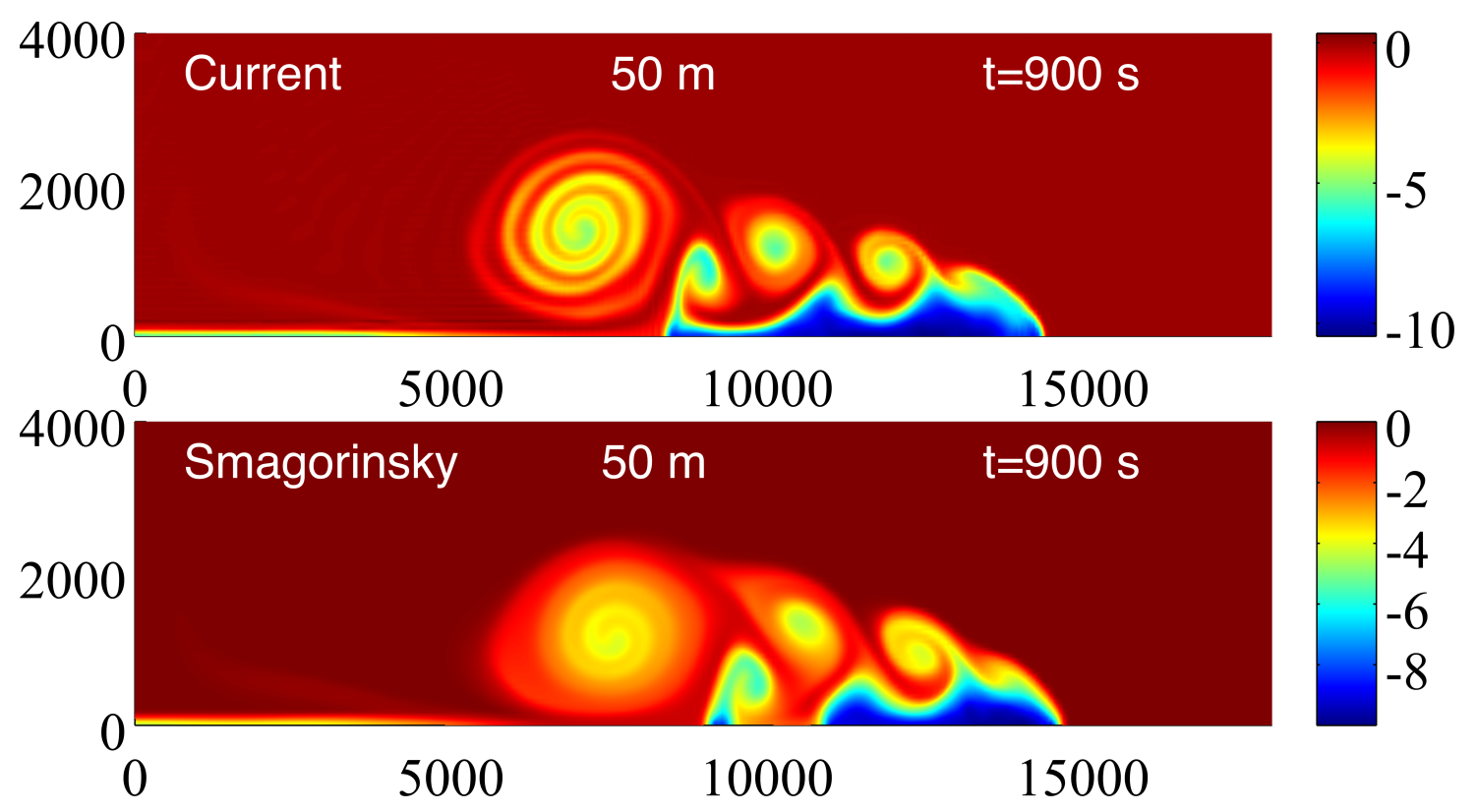

Figure 6: $2 \frac{1}{2} \mathrm{D}$ density current. Comparison of the current method (top) with the solution obtained using Smagorinsky with constant $C_{s}=0.14$ (bottom) using a grid resolution $\Delta x=\Delta z=50 \mathrm{~m}$.

is viscous; free-slip boundary conditions are applied on every solid wall. This is an important point since it is well known that Smagorinsky is overly dissipative in boundary layer flows unless it is properly corrected. We are currently working on a thorough comparison of three LES models that are commonly used in atmospheric simulations and the results will be reported in a subsequent paper.

This test case does not admit an analytic solution. To compare with other models we look at the front position at $t=900 \mathrm{~s}$, where the front is defined as the position on the ground where $\Delta \theta=-1 \mathrm{~K}$. In Table 3, the position of the front is reported for Dyn-SGS, for our implementation of Smagorinsky, and for some of the results reported in the literature. As the grid is coarsened, the front is slightly slower; this fact is also observed in Fig. 5 of [48]. On the $50 \mathrm{~m}$ grid, all models agree with a front location in the range $[14409,14975] \mathrm{m}$. For the data that are available at different resolutions (see current, Smagorinsky, and VMS in the table), a trend is observed: as the resolution is increased from $200 \mathrm{~m}$ to $12.5 \mathrm{~m}$, the front moves relatively faster although within a few meters of difference in the front position from the fine to the coarse grid solution. The somewhat smaller speed in the case of a coarser grid is to be attributed to the relatively larger dissipation in the coarse solution; this makes the flow more viscous and hence gives it a tendency to be slightly slower.

As the resolution is increased, the amount of structures that are resolved increases as well. Without the large viscosity that homogenizes the solution as done in [48] with the sole target of reaching convergence, the inviscid, non-linear, and non-steady solution that we present here is not expected to show signs of space-convergence. The same behavior was observed in [41], where VMS was used to stabilize a finite element discretization of the Euler equations. Rather, we expect more and more structures to be resolved until a grid resolution of the order of the smallest eddies is 


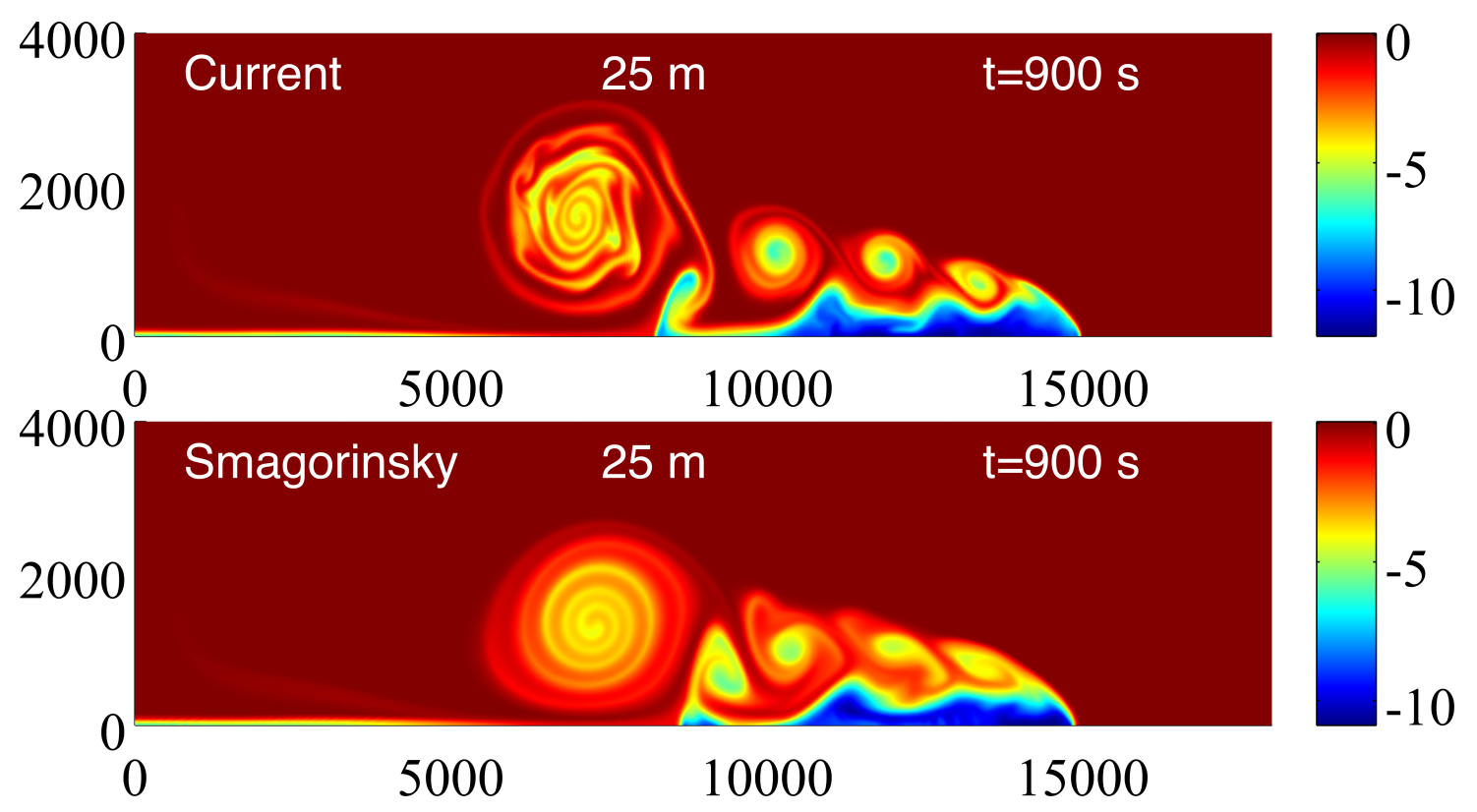

Figure 7: As in Fig. 6, but at the finer resolution $\Delta x=\Delta z=25 \mathrm{~m}$.

reached.

\subsection{D warm bubble}

So far only $2 \frac{1}{2} \mathrm{D}$ problems have been presented, where the solution was sought in a $3 \mathrm{D}$ domain with infinite extension along the $y$-direction. To test the complete applicability of the current method to fully three-dimensional problems, we consider the $3 \mathrm{D}$ analog of the $2 \frac{1}{2} \mathrm{D}$ buoyant rising thermal described above. The problem is now solved in the domain $\Omega=[0,1000]^{3} \mathrm{~m}^{3}$ as in [33]. The initial perturbation $\Delta \theta$ is no longer linear; it is given by the function

$$
\Delta \theta=\frac{\theta_{c}}{2}\left[1.0+\cos \left(\frac{\pi r}{r_{0}}\right)\right] \quad \text { if } r \leq r_{0}=250 \mathrm{~m},
$$

where $r=\sqrt{\left(x-x_{c}\right)^{2}+\left(y-y_{c}\right)^{2}+\left(z-z_{c}\right)^{2}},\left(x_{c}, y_{c}, z_{c}\right)=(500,500,260) \mathrm{m}$, and $\theta_{c}=0.5 \mathrm{~K}$. To verify the preservation of the axial symmetry of the problem, we solve the fully $3 \mathrm{D}$ problem without relying on its axial symmetry. In Figs. 8 and 9 , the $4^{\text {th }}$ and, respectively, $8^{\text {th }}$-order solutions are shown at $t=400 \mathrm{~s}$. With $n_{e}=10 \times 10 \times 10$, the effective resolutions are 25 and $12.5 \mathrm{~m}$. Based on [33], a constant and uniform artificial diffusion (indicated by the symbol $H V_{2}$ ) is expected to lead to a solution that varies according to the value of its coefficient. The current method is designed to dissipate the solution only where necessary. The value of the extrema of the current solution is in close agreement with the $H V_{2}$ solution using $0.5 \mathrm{~m}^{2} \mathrm{~s}^{-1}(\max (\Delta \theta) \approx 0.35)$, indicating that the amount of diffusion that is being added for stabilization is sufficiently small to not dissipate the solution away from the region of larger residuals, but is large enough to suppress all the oscillations 
Table 3: Density current. Comparative results of front location at $t=900 \mathrm{~s}$. The results are reported for the following models: current model, Smagorinsky implemented in our code, VMS, WRF-ARW, $f$-wave, filtered Spectral Elements (SE), filtered Discontinuous Galerkin (DG), REFC, REFQ, and PPM. All models but the current, Lilly-Smagorinsky, and VMS, used artificial diffusion with constant $\mu=75 \mathrm{~m}^{2} \mathrm{~s}^{-1}$.

\begin{tabular}{|c|c|c|c|c|c|c|}
\hline Model & Space discr. & Resolution & $n_{e}$ & Order & $\mu=75 \mathrm{~m}^{2} \mathrm{~s}^{-1}$ & Front Location $[\mathrm{m}]$ \\
\hline Current (Dyn-SGS) & SEM & $12.5 \mathrm{~m}$ & $512 \times 1 \times 128$ & $4^{t h}$ & No & 15056 \\
\hline " & $"$ & $25 \mathrm{~m}$ & $256 \times 1 \times 64$ & $4^{t h}$ & No & 14992 \\
\hline " & $"$ & $50 \mathrm{~m}$ & $128 \times 1 \times 32$ & $4^{t h}$ & No & 14535 \\
\hline " & $"$ & $100 \mathrm{~m}$ & $64 \times 1 \times 16$ & $4^{t h}$ & No & 14325 \\
\hline " & $"$ & $200 \mathrm{~m}$ & $32 \times 1 \times 8$ & $4^{t h}$ & No & 13552 \\
\hline " & $"$ & $133 \mathrm{~m}$ & $32 \times 1 \times 8$ & $6^{t h}$ & No & 14568 \\
\hline " & $"$ & $100 \mathrm{~m}$ & $32 \times 1 \times 8$ & $8^{t h}$ & No & 14754 \\
\hline Smagorinsky & SEM & $25 \mathrm{~m}$ & $256 \times 1 \times 64$ & $4^{t h}$ & No & 14918 \\
\hline " & " & $50 \mathrm{~m}$ & $128 \times 1 \times 32$ & $4^{t h}$ & No & 14726 \\
\hline " & $"$ & $100 \mathrm{~m}$ & $64 \times 1 \times 16$ & $4^{t h}$ & No & 14551 \\
\hline VMS [41] & FEM & $25 \mathrm{~m}$ & & & No & 14890 \\
\hline$"[41]$ & " & $50 \mathrm{~m}$ & & & No & 14629 \\
\hline " [41] & " & $75 \mathrm{~m}$ & & & No & 14487 \\
\hline " [41] & $"$ & $100 \mathrm{~m}$ & & & No & 14355 \\
\hline SE [20] & SEM & $50 \mathrm{~m}$ & & & Yes & 14767 \\
\hline DG $[20]$ & DG & $50 \mathrm{~m}$ & & & Yes & 14767 \\
\hline$f$-wave $[2]$ & FV & $50 \mathrm{~m}$ & & & Yes & 14975 \\
\hline WRF-ARW $[2$, run in- $]$ & FD & $50 \mathrm{~m}$ & & & Yes & 14470 \\
\hline REFC [48] & FD & $50 \mathrm{~m}$ & & & Yes & 14437 \\
\hline REFQ [48] & FD & $50 \mathrm{~m}$ & & & Yes & 14409 \\
\hline PPM [48] & FD & $50 \mathrm{~m}$ & & & Yes & 15027 \\
\hline
\end{tabular}

that are visible in the case of $H V_{2}$. As previously observed for the density current, the higher the resolution the faster the front. Although the difference in height is minimal, it is still noticeable within a few meters difference. This same test was run in [33] using an effective resolution of $12.5 \mathrm{~m}$ $\left(8^{t h}\right.$-order) and in [19] with an effective resolution of $10 \mathrm{~m}$. In the latter, the front is approximately $50 \mathrm{~m}$ higher than it is in the case of [19]. This said, the current method produces a thermal whose dissipation is as limited and constrained in space as it is for $H V_{2}\left(0.5 \mathrm{~m}^{2} \mathrm{~s}^{-1}\right)$ but, at the same time, preserves the smoothness of $H V_{2}\left(5.0 \mathrm{~m}^{2} \mathrm{~s}^{-1}\right)$. The $4^{\text {th }}$ and $8^{t h}$-order solutions show the same behavior. It is also shown that its stability properties are not compromised or modified as the order of the spectral element is increased from 4 to 8 . It is important to keep this in mind since the increase of the element order coincides with an important decrease in the grid spacing within each element, and the current version of the stabilizing method is a function of the element effective resolution.

\subsection{Baroclinic-wave in a 3D channel with geostrophically balanced back- ground}

A geostrophically balanced background is defined as in [51]. The $f$-plane approximation is considered. The flow is confined in a very-high aspect ratio domain $\Omega=\left[L_{x} \times L_{y} \times L_{z}\right]=[40000 \times 6000 \times 30] \mathrm{km}^{3}$ with periodic boundary conditions along the flow direction and free-slip, non-penetrating boundaries everywhere else. Notice how the characteristic size of this domain (and of the grid spacing) 

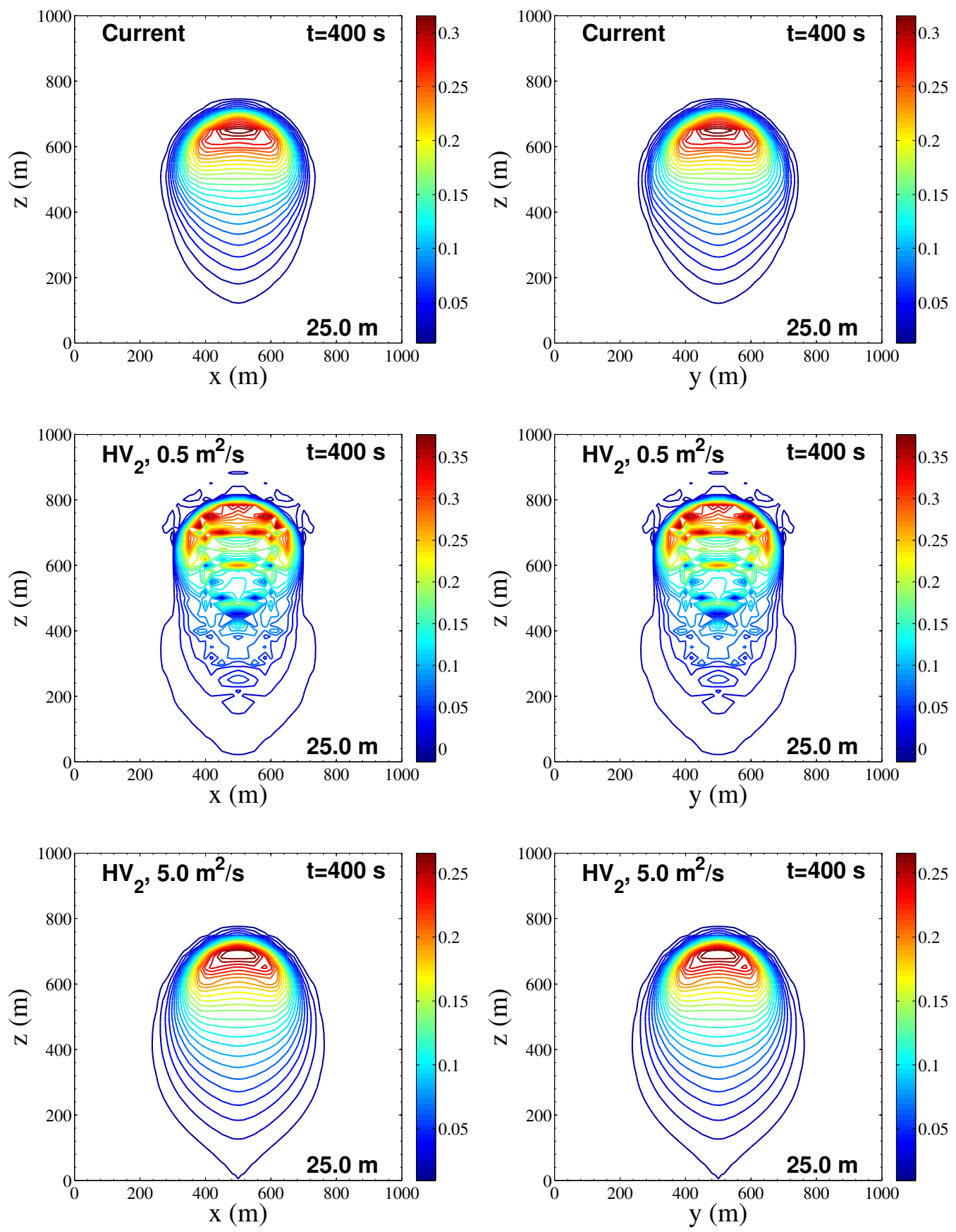

Figure 8: 3D rising thermal bubble. Solution at $t=400 \mathrm{~s}$. Left column: $x z$-slice of $\Delta \theta$ at $y=500$ m. Right column: $y z$-slice at $x=500 \mathrm{~m}$. Top row: solution using the current method (Dyn-SGS). Middle row: solution using second-order hyper diffusion $\left(H V_{2}\right)$ with constant coefficient $\mu=0.5$. Bottom row: second-order hyper diffusion with constant coefficient $\mu=5$.0. Courant number 20.0 on a $25 \mathrm{~m}$ effective resolution grid of order $4 . \Omega=[1000]^{3} \mathrm{~m}^{3}$. 

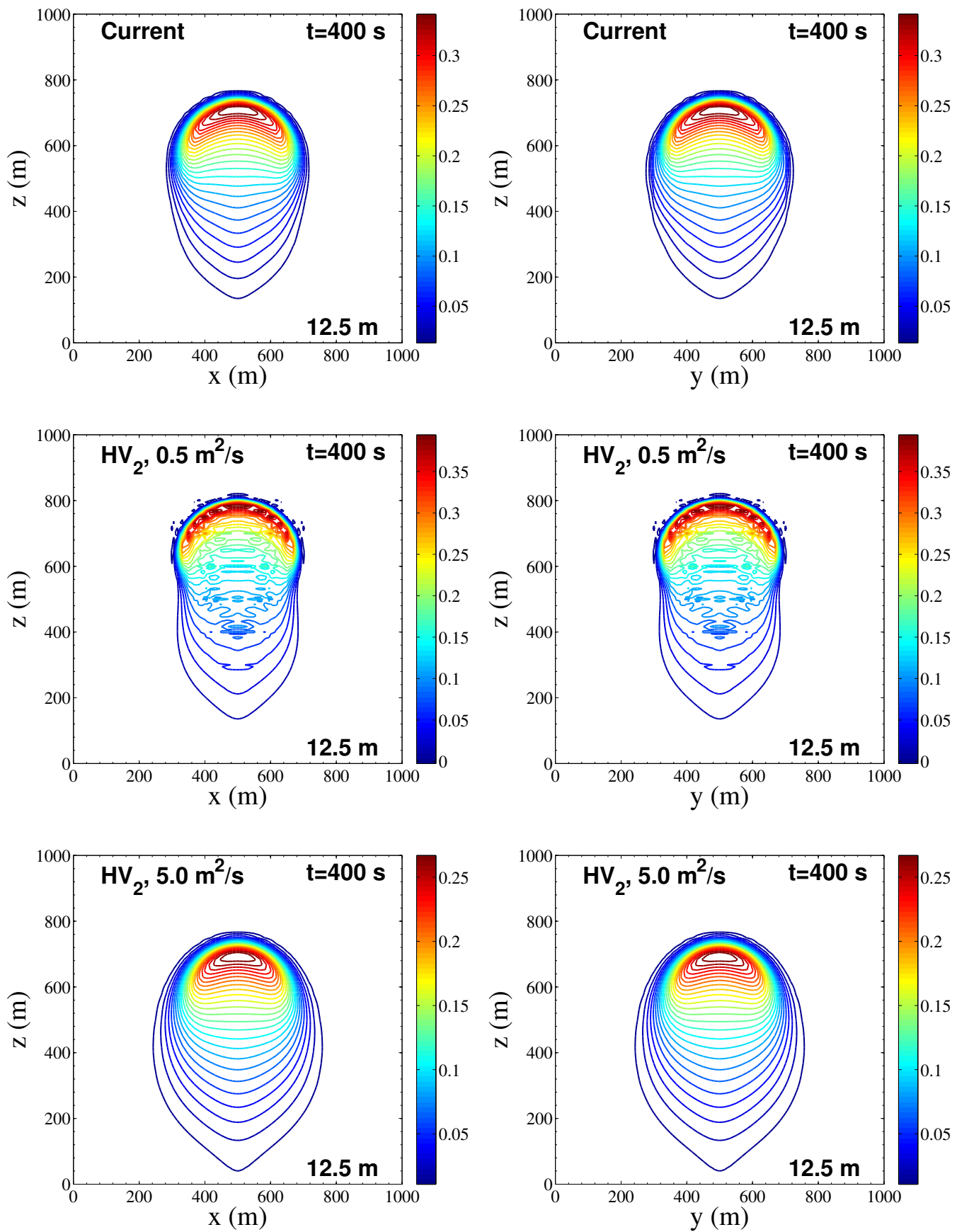

Figure 9: As Fig. 8, but for $8^{t h}$-order elements and effective resolution of $12.5 \mathrm{~m}$, and Courant number 17.5 . 
is up to $\mathcal{O}\left(10^{4}\right)$ times larger than that of the previous problems. If we consider a pressure-based vertical coordinate $\eta$, from which the $z$ coordinate is derived via iteration (see appendix in [30]), the initial jet is a zonally symmetric flow defined as

$$
u(x, y, \eta)=-u_{0} \sin ^{2}\left(\frac{\pi y}{L_{y}}\right) \ln \eta \exp \left[-\left(\frac{\ln \eta}{b}\right)^{2}\right],
$$

with amplitude $u_{0}=35 \mathrm{~ms}^{-1}$ and vertical width parameter $b=2$. The meridional and vertical velocities, $(v, w)$, are initially zero. In the $f$-plane approximation the Coriolis parameter is $f_{0}=$ $2 \omega \sin \varphi_{0}$ at the latitude $\varphi_{0}=45^{\circ} \mathrm{N}$, where $\omega$ is the Earth rotational velocity (Table 1 ). Although in a plane channel, this jet is designed to resemble a mid-latitude westerly zonal wind with a zonaland time-mean jet speed at the earth troposphere. The background geopotential is given by

$$
\Phi(x, y, \eta)=\frac{T_{0} g}{\Gamma}\left(1-\eta^{R_{d} \Gamma / g}\right)+\Delta \Phi(x, y) \ln \eta \exp \left[-\left(\frac{\ln \eta}{b}\right)^{2}\right],
$$

where $T_{0}=288 \mathrm{~K}$ is a reference temperature, $\Gamma=0.005 \mathrm{Km}^{-1}$ is the lapse rate, and $\Delta \Phi$ is the perturbation of $\Phi$ given by

$$
\Delta \Phi=\frac{u_{0}}{2} f_{0}\left[y-\frac{L_{y}}{2}-\frac{L_{y}}{2 \pi} \sin \left(\frac{2 \pi y}{L_{y}}\right)\right] .
$$

The perturbed temperature distribution is given by

$$
T(x, y, \eta)=T_{0} \eta^{R_{d} \Gamma / g}+\frac{\Delta \Phi(x, y)}{R}\left(\frac{2}{b^{2}} \ln ^{2} \eta-1\right) \exp \left[-\left(\frac{\ln \eta}{b}\right)^{2}\right] .
$$

Baroclinic instabilities are responsible for mid-latitude cyclones [26] and are thus important atmospheric processes for an atmospheric model to capture. The baroclinic wave instability is triggered by a perturbation of the initially balanced zonal velocity field. As the wave breaks, gravity waves are radiated with the intent of restoring the initial geostrophic balance [25]. The perturbation is given by an unbalanced smooth profile centered at $\left(x_{c}, y_{c}\right)=(2000,2500) \mathrm{km}$ and defined as

$$
\Delta u(x, y, z)=u_{p} \exp \left[-\frac{\left(x-x_{c}\right)^{2}+\left(y-y_{c}\right)^{2}}{L_{p}^{2}}\right],
$$

where $u_{p}=1 \mathrm{~ms}^{-1}$ is the perturbation amplitude and $L_{p}=600 \mathrm{~km}$ is the width parameter.

We have seen above how the dynamic dissipation depends on a characteristic grid size. Due to the very high aspect ratio of the current grids, with the vertical resolution being 100 times smaller than the horizontal one, stabilization for this problem was first run by setting the vertical diffusion to a value 100 times smaller along $z$ than it is along $x$ and $y$, and then simply to zero along $z$. The result did not change significantly so that the plots shown in this paper are only those obtained with zero vertical dissipation. The vertical resolution is sufficiently high to preserve stability without the need for vertical diffusion.

To get a sense of the error that we commit and compare against [51], we first ran this test without perturbing the initially balanced flow. The relative error norms $L_{1}, L_{2}$, and $L_{\infty}$ of $\mathrm{q}=\rho \theta$ are defined as

$$
L_{1}(q(\mathbf{x}, t))=\frac{\int_{\Omega^{h}}|q(\mathbf{x}, t)-q(0, \mathbf{x})| d \Omega^{h}}{\int_{\Omega^{h}}|q(0, \mathbf{x})| d \Omega^{h}},
$$


Table 4: Error norms of $\rho \theta$ in the solution of the geostrophically balanced flow in a 3D channel for 5 different horizontal resolutions, but the same $1 \mathrm{~km}$ vertical grid spacing. The errors are computed at day 1 with respect to the initial condition. For comparison, the errors of the non-viscous solution are also reported and are indicated by the $\hat{\cdot}$ symbol.

\begin{tabular}{lcc|cc|cc}
\hline \hline & & & & & \\
$\mathrm{km}$ & $L_{1}$ & $\widehat{L_{1}}$ & $L_{2}$ & $\widehat{L_{2}}$ & $L_{\infty}$ & $\widehat{L_{\infty}}$ \\
\hline 800 & $2.77 \times 10^{-5}$ & $2.75 \times 10^{-5}$ & $7.44 \times 10^{-10}$ & $7.26 \times 10^{-10}$ & $4.27 \times 10^{-5}$ & $4.19 \times 10^{-5}$ \\
400 & $3.11 \times 10^{-6}$ & $3.11 \times 10^{-6}$ & $1.14 \times 10^{-11}$ & $1.14 \times 10^{-11}$ & $6.91 \times 10^{-6}$ & $6.91 \times 10^{-6}$ \\
200 & $8.81 \times 10^{-8}$ & $8.81 \times 10^{-8}$ & $6.27 \times 10^{-15}$ & $9.27 \times 10^{-15}$ & $4.03 \times 10^{-7}$ & $4.03 \times 10^{-7}$ \\
100 & $7.21 \times 10^{-9}$ & $7.21 \times 10^{-9}$ & $5.35 \times 10^{-17}$ & $5.35 \times 10^{-17}$ & $1.78 \times 10^{-8}$ & $1.78 \times 10^{-8}$ \\
50 & $6.26 \times 10^{-10}$ & $6.25 \times 10^{-10}$ & $4.16 \times 10^{-19}$ & $4.16 \times 10^{-19}$ & $1.24 \times 10^{-9}$ & $1.24 \times 10^{-9}$ \\
\hline \hline
\end{tabular}

$$
L_{2}(q(\mathbf{x}, t))=\sqrt{\frac{\int_{\Omega^{h}}(q(\mathbf{x}, t)-q(0, \mathbf{x}))^{2} d \Omega^{h}}{\int_{\Omega^{h}} q^{2}(0, \mathbf{x}) d \Omega^{h}}},
$$

and

$$
L_{\infty}(q(\mathbf{x}, t))=\frac{\max |q(\mathbf{x}, t)-q(0, \mathbf{x})|}{\max |q(0, \mathbf{x})|},
$$

where $q(0, \mathbf{x})$ is the initial solution and the integrals are computed via the usual quadrature formulas. We report their values at day 1 in Table 4 . The errors obtained with stabilization are compared against our inviscid solution and against the inviscid solution of [51]. Because this problem is smooth and the flow is stationary, the dissipative effect of the current method should be negligible. Although some small dissipation is still partially active across the whole domain (plot of the SGS is not shown for this case), its effect is indeed minor, as can be seen by the almost non-existent difference between the tabulated errors of the stabilized and inviscid solutions. These errors are plotted in Fig. 10 as well; the error decay shows that the order of the numerical approximation is not degraded. In the same figure, the time evolution of the error is plotted for a 20 day simulation.

In the case of the perturbed flow that triggers a baroclinic wave, the evolution of $T$ from day 12 to day 14 is shown in Fig. 11 given an anisotropic grid with $\Delta x=100 \mathrm{~km}, \Delta y=75 \mathrm{~km}, \Delta z=1.25$ $\mathrm{km}$. (Note: to our knowledge, no result has been shown past day 12. We believe an instability in the problem statement is responsible.) The plots show the solution on the $x y$ cross section at $z=500$ $\mathrm{m}$. With respect to the results of [51], the wave breaking occurs at the same time (approximately 9 days). The values of $\mathrm{T}$ and of the vertical component of vorticity are in agreement with [51] at all resolutions. The flow at $t>12$ days is shown as well for future comparisons.

\subsection{Mass loss}

Next, we wish to analyze the effect of Dyn-SGS on the mass conservation since this is an important metric for atmospheric models. Let us define the time dependent normalized mass loss as

$$
M(t)_{l o s s}=\frac{\int_{\Omega}\left[\bar{\rho}(t)-\bar{\rho}\left(t_{0}\right)\right] d \Omega}{\int_{\Omega} \bar{\rho}\left(t_{0}\right) d \Omega},
$$

where $\bar{\rho}\left(t_{0}\right)$ indicates density at the initial time. Figure 12 shows $M(t)_{l o s s}$ for a $20400 \mathrm{~s}$ simulation of the $2 \frac{1}{2} \mathrm{D}$ rising thermal bubble at three different resolutions and three stabilization methods: the current one, a $2^{\text {nd }}$-order artificial diffusion, $H V_{2}$ with constant coefficient, and a $4^{\text {th }}$-order hyper 

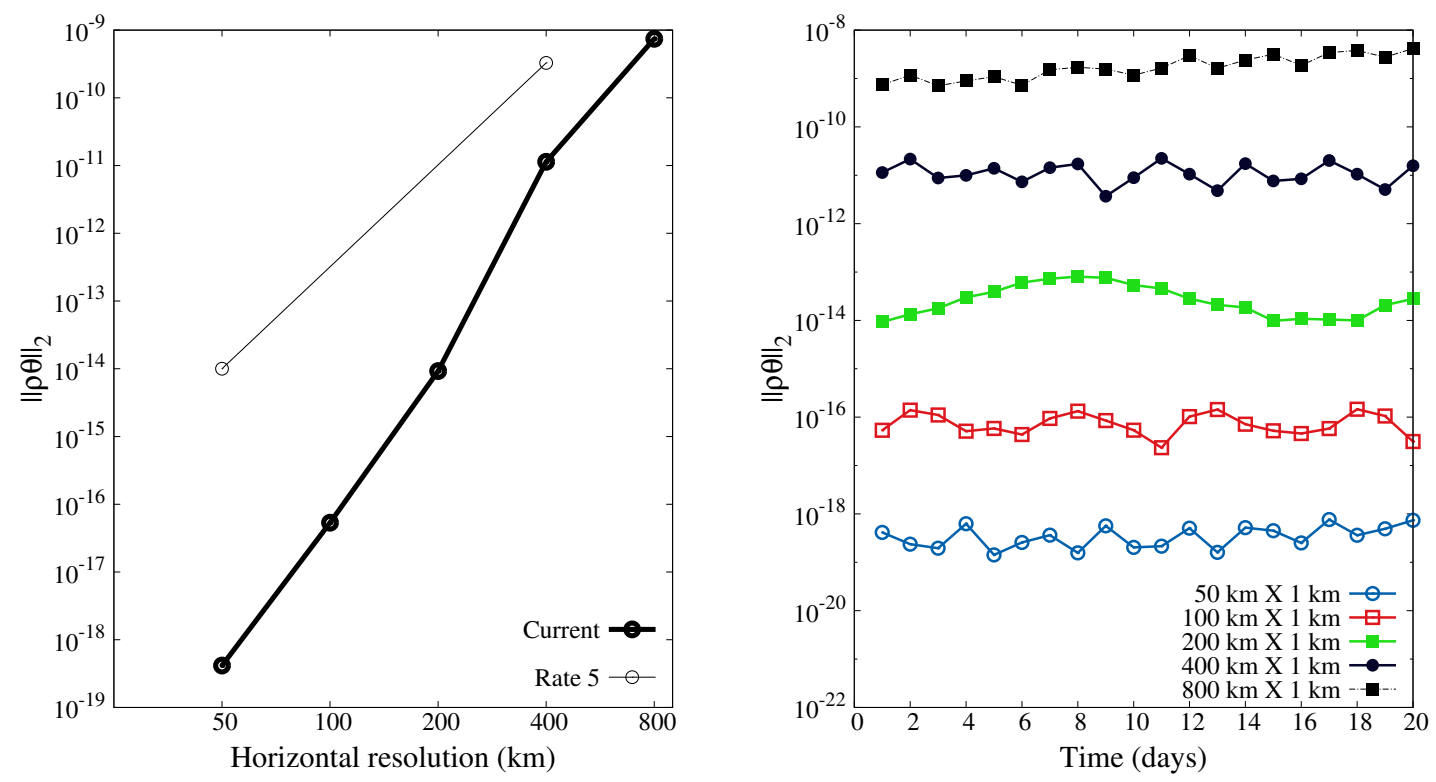

Figure 10: Normalized $h$-error norms for the solution of the geostrophically balanced flow in a 3D channel. Left plot: grid convergence (log-log scale). Right: time evolution of $\|\rho \theta\|_{2}$.

diffusion, $H V_{4}$, with constant coefficient. All methods show a trend towards higher mass loss values as the resolution is increased. For instance, the current case has the lowest value that approximates machine precision in the case of 85 and $170 \mathrm{~m}$ resolutions but increases to higher values in the case of the $45 \mathrm{~m}$ resolution. In the case of $H V_{2}$ and $H V_{4}$, the mass loss is always in the proximity of $1 \times 10^{-14}$, although the curves are visibly higher for higher resolutions. In spite of the differences among the three methods, a sustained mass loss $\mathcal{O}\left(\leq 10^{-14}\right)$ is an acceptable value. At the coarsest resolution of $170 \mathrm{~m}, H V_{4}$ failed to preserve stability with the given coefficient; this is why the time series is truncated at approximately 16000 seconds. Although a different coefficient would immediately solve this problem, the search for a better coefficient falls beyond the scope of this paper.

In Fig. 13, we plot the mass loss for a 20 day simulation of the geostrophically balanced flow in a $3 \mathrm{D}$ channel. The very large time scale of this problem is a good test for mass conservation in a geophysical flow. Although not at machine accuracy and in spite of a light tendency towards mass increase that begins at approximately day $6, \mathcal{O}\left(1 \times 10^{-15}\right)$ mass loss is still indicating that the method is, in this respect, robust for very long simulations. A significant mass loss increase or oscillation would be indicative of an improper behavior of the model.

\section{Model verification: quasi-linear scalar equations}

We test the model against the solution of the quasi-linear 1D Burgers equation. The reason for including this test is twofold: first, we demonstrate that Dyn-SGS can also be used to stabilize scalar equations, which are typically required as part of atmospheric modeling systems; second, this problem admits an exact solution to compare against. The solution of linear transport problems 

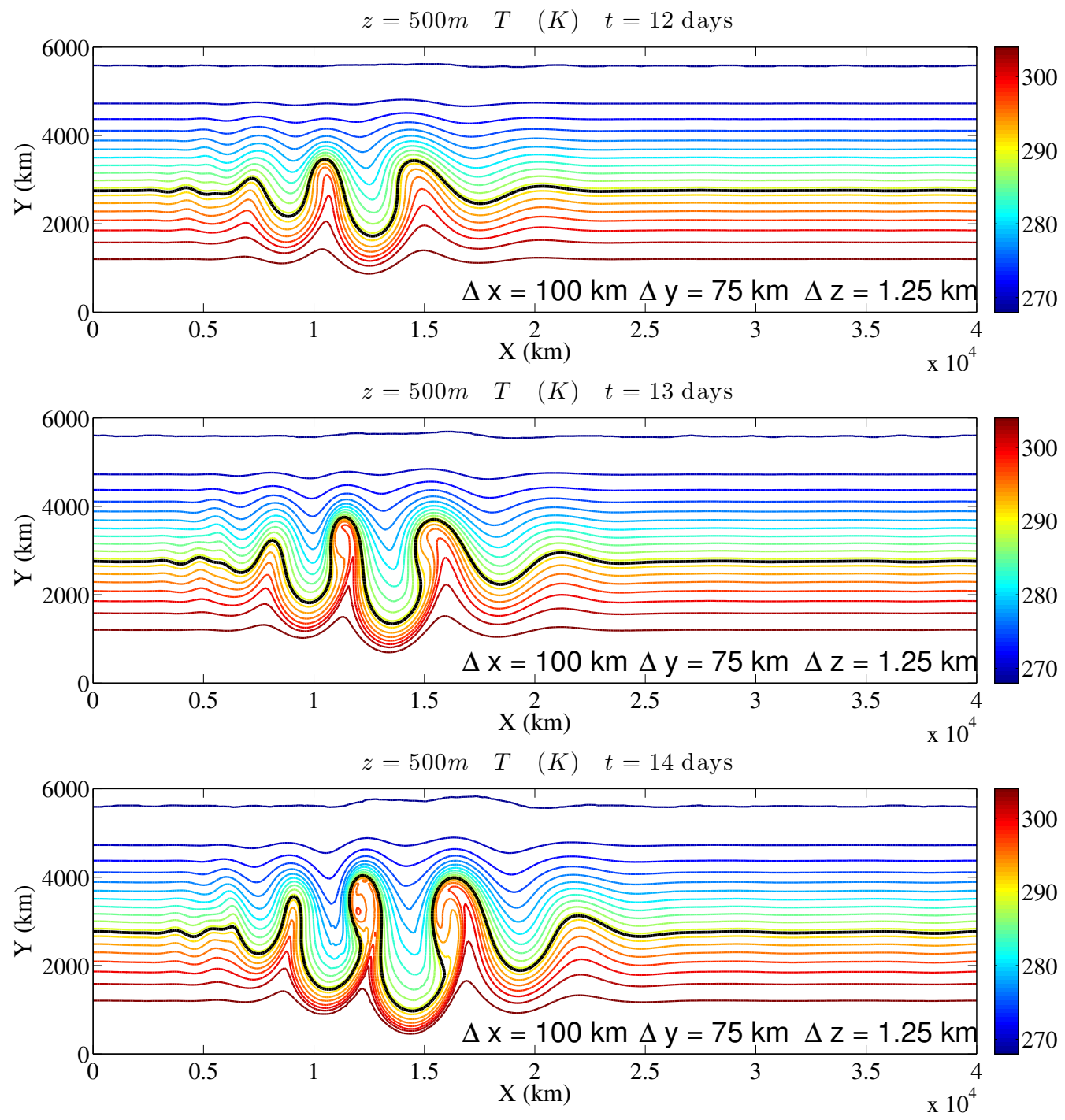

Figure 11: Baroclinic instability in a channel. $f$-plane solution at $z=500 \mathrm{~m}$. The temperature is plotted at days $12,13,14$ on a grid resolution $\Delta x=100 \mathrm{~km}, \Delta y=75 \mathrm{~km}, \Delta z=1.25 \mathrm{~km}$ using $4^{\text {th }}$-order polynomials. The thick contour corresponds to $T=290 \mathrm{~K}$. 


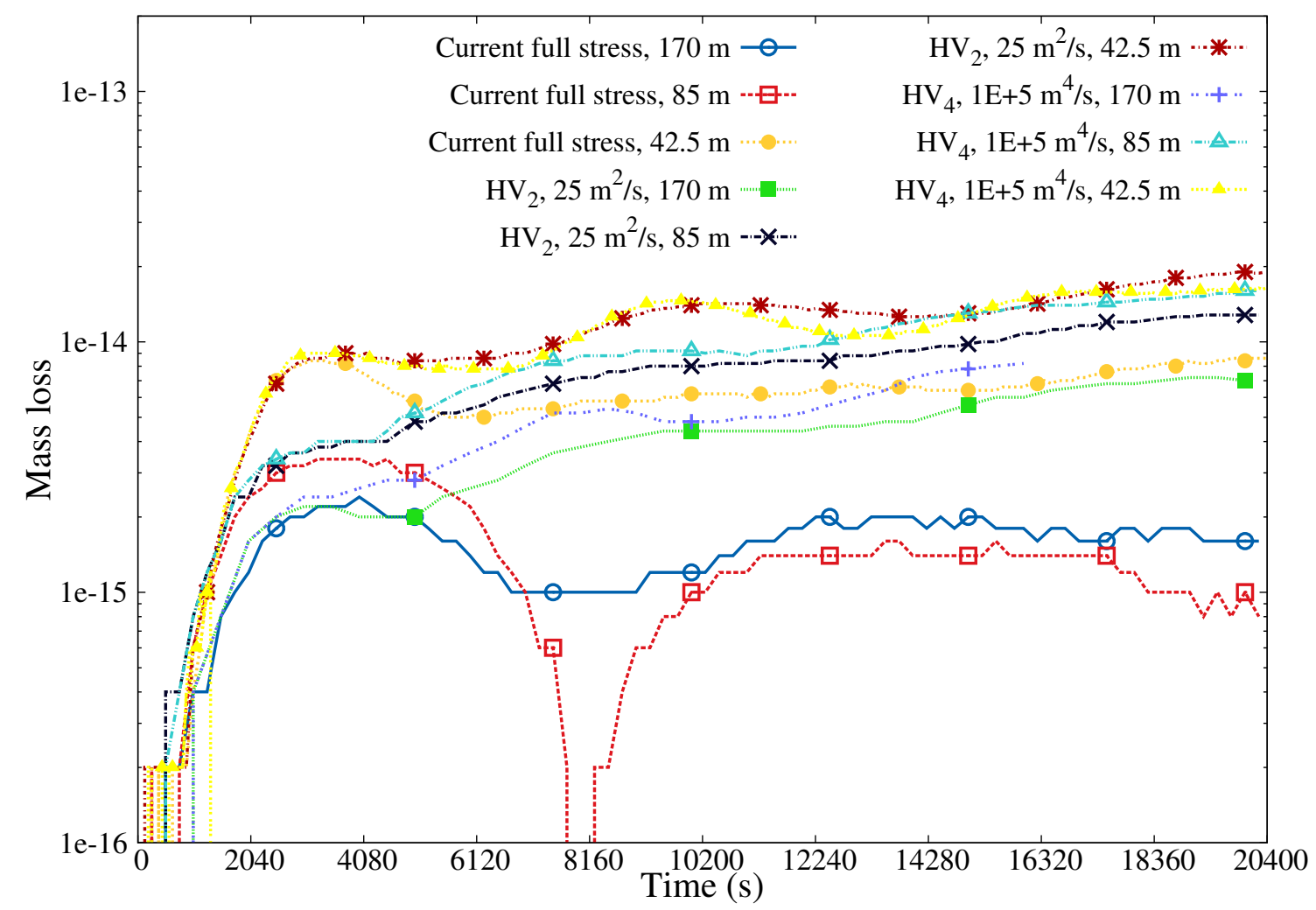

Figure 12: Time evolution of the mass loss for the stabilized solution using the current method, using $H V_{2}$ with constant coefficient $\nu=25 \mathrm{~m}^{2} \mathrm{~s}^{-1}$, and using $H V_{4}$ with $\nu=1 E+5 \mathrm{~m}^{4} \mathrm{~s}^{-1}$ in the simulation of the $2 \frac{1}{2} \mathrm{D}$ rising thermal bubble. All methods show a trend towards higher mass loss values as the resolution is increased. For instance, the current case has the lowest value that approximates machine precision in the case of 85 and 170 meter resolutions, but increases to higher values at $45 \mathrm{~m}$ resolution. In the case of $H V_{2}$ and $H V_{4}$, the mass loss is always in the proximity of $1 \times 10^{-14}$, although the curves are visibly higher at higher resolution. At the coarsest resolution of $170 \mathrm{~m}, H V_{4}$ failed to preserve stability with the given coefficient; this is why the time series is truncated at approximately 16000 seconds. A different coefficient would immediately solve this problem, although its search is not relevant in the context of this paper. 


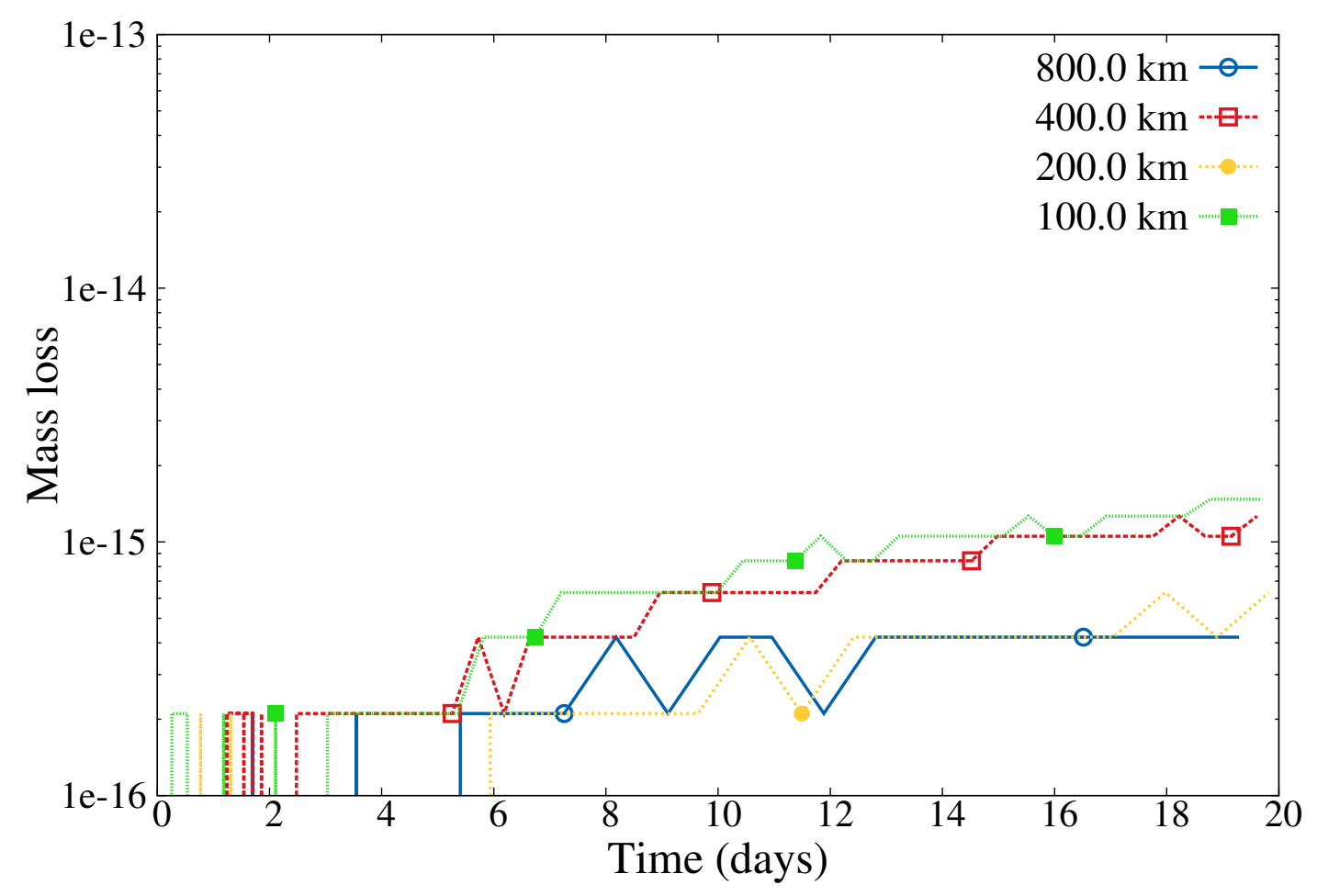

Figure 13: Time evolution of the mass loss for the stabilized solution of the geostrophically balanced flow in a $3 \mathrm{D}$ channel. 
via Dyn-SGS is described in a recent report [39].

The solution is computed in $\Omega(x)=[0,2]$ for the initial condition $u(x, 0)=0.5+\sin (\pi x)$ and periodic boundary conditions. The solution is advanced in time until $t=2 / \pi$. The solution is computed on a 40 and on an 80 element grid of order 8 , which correspond to the effective resolutions $\Delta x=6.25 \times 10^{-3} \mathrm{~m}$ and $\Delta x=3.125 \times 10^{-3} \mathrm{~m}$, respectively. The exact solution of the inviscid Burgers equation is computed via the method of characteristics. The characteristic curves of the computed solution are plotted in Fig. 14. As time approaches $1 / \pi \mathrm{s}$, the characteristic curves begin to cross, generating a shock that becomes stronger as time passes. At all times, the solution remains smooth sufficiently far from the shock, as visible from the point-wise relative error, $\left|u-u_{\text {exact }}\right|$, plotted in Fig. 15 on the $x-t$ plane. The superposition of the computed and exact solutions at the two time levels $t=[3 /(2 \pi), 2 / \pi]$ is plotted in Figs. 16 and, respectively, 17 . The shock is well captured within one element. In spite of the high-order approximation $\left(8^{t h}\right.$-order elements), the over- and under-shoots in the proximity of the shock are limited, very well controlled, and do not propagate from the shock across the domain. This is confirmed by the point-wise error curves plotted in Fig. 18. Our results agree with the entropy-viscosity solution of [22]. Moreover, the sharpness of the discontinuous solution seems uncompromised by the action of the dynamic diffusion. This is a strong result that demonstrates well the shock-capturing capabilities of Dyn-SGS when high-order methods are used.

Figure 19 shows the element-wise structure of $\mu$, Eq. (15), at $t=[1 / \pi, 3 /(2 \pi), 2 / \pi]$ s. $\mu$ has no effect on the solution in the smooth regions, as it clearly acts only in the neighborhood of the shock.

The normalized $L_{1}, L_{2}$, and $L_{\infty}$ error norms are plotted in Fig. 20 as a function of time. As expected, the formation of the shock has a major effect on the accuracy of the solution, as is visible from the jump in the infinity norm.

\section{Conclusions}

We presented the application of a dynamic sub-grid scale model (Dyn-SGS) for Large Eddy Simulation to stabilize the spectral element (SEM) solution of low Mach number stratified flows and of quasi-linear scalar transport. Possibly, the most important features of Dyn-SGS that emerged from this study are the following:

- For smooth problems, this model does not deteriorate the nominal order of accuracy of the spectral approximation of the governing equations (see the error norms computed for the geostrophically balanced flow in a 3D channel.)

- It is flexible and robust with respect to the flow regime and grid size.

- It is completely free of a user-tunable parameter.

- In the neighborhood of sharp gradients, it limits and controls the magnitude of the over- and under-shoots without compromising the solution away from the discontinuity. Moreover, the sharpness of the discontinuity is very well preserved.

It was also shown that dynamic stabilization and large eddy simulations are achieved by one scheme alone.

When it comes to parallel performance, the cost of this method is that of a second-order Laplace operator, whose computation only requires one communication, against the two (or more) necessary 

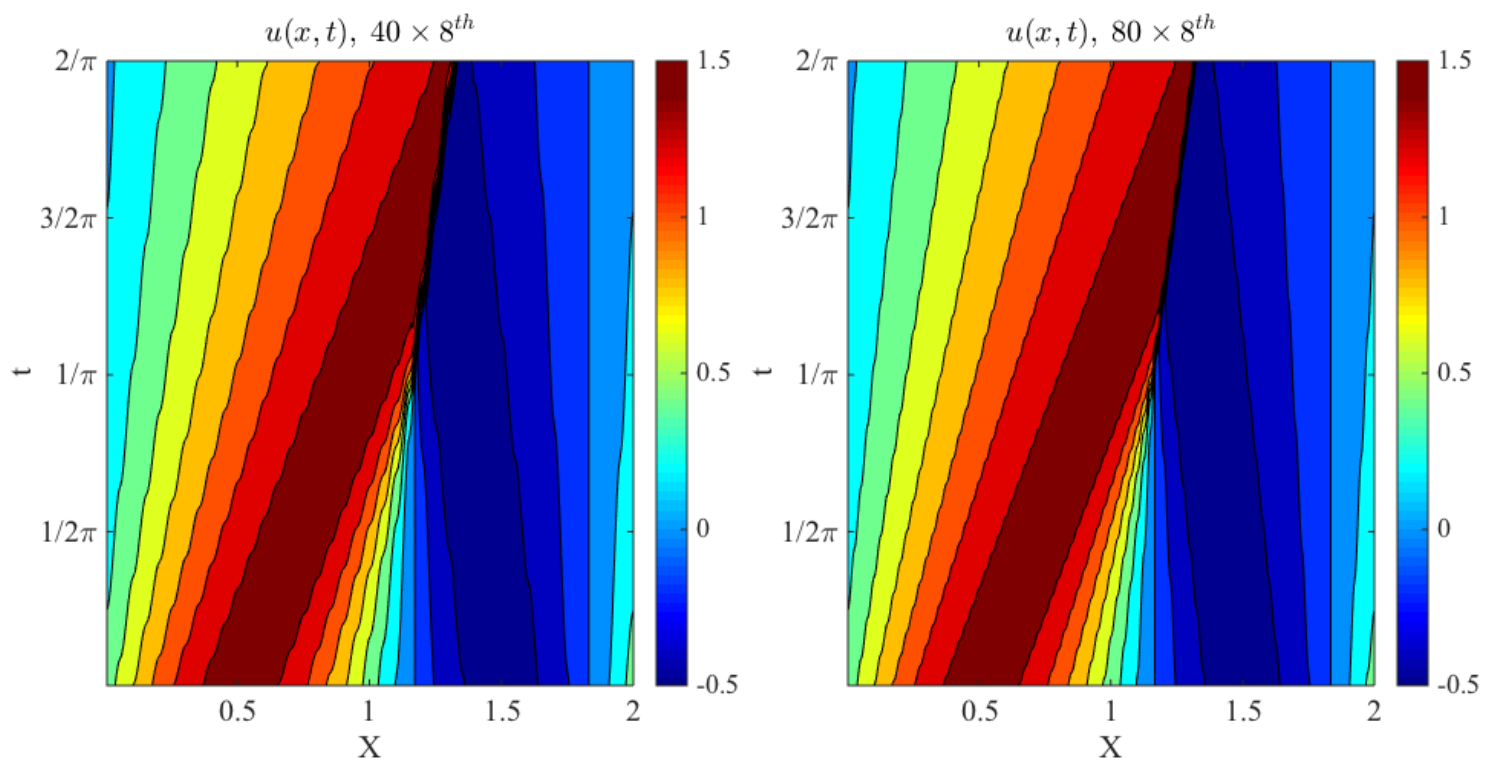

Figure 14: 1D Burgers equation: Characteristic curves of the computed solution. Left: $\Delta x=$ $6.25 \times 10^{-3} \mathrm{~m}$. Right: $\Delta x=3.125 \times 10^{-3} \mathrm{~m}$.
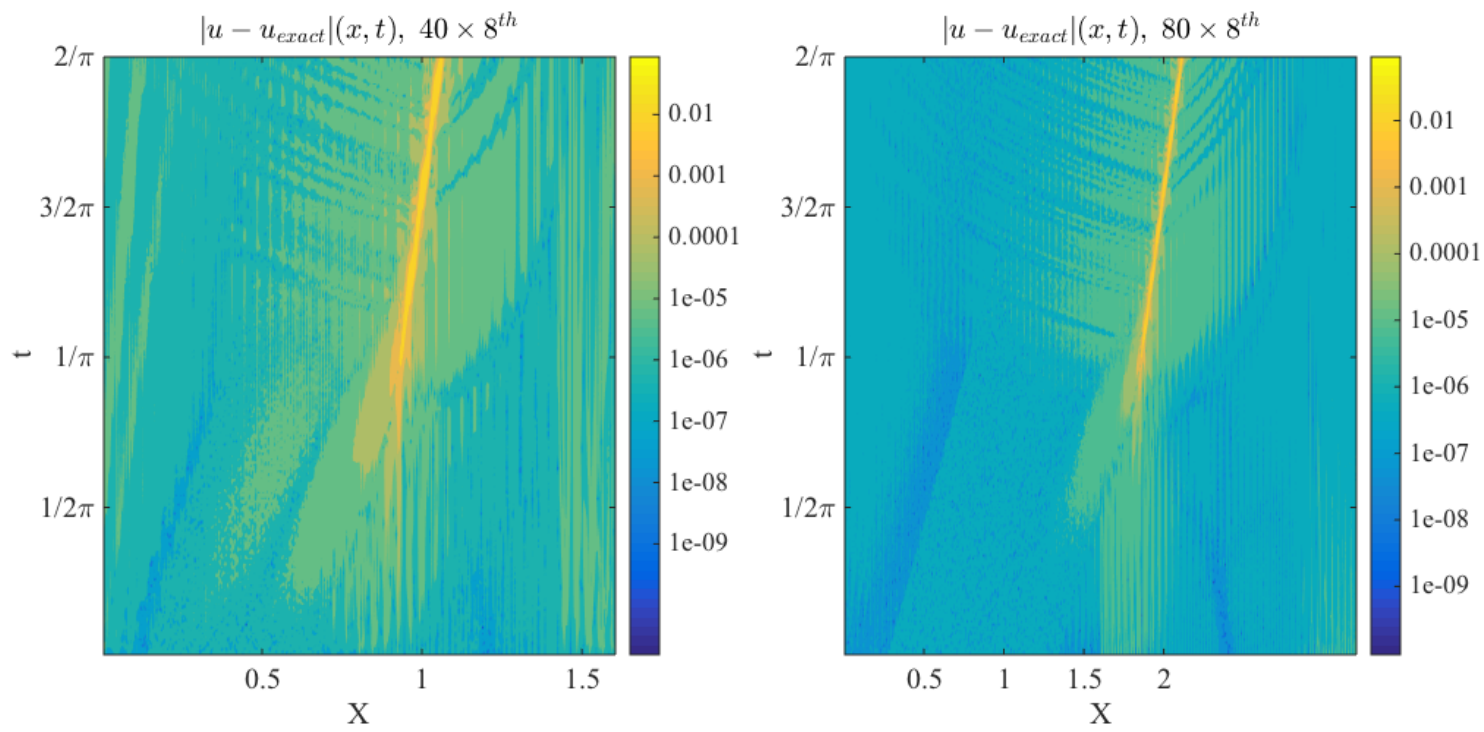

Figure 15: 1D Burgers equation. Point-wise relative error in logarithmic scale on the $x-t$ plane. 

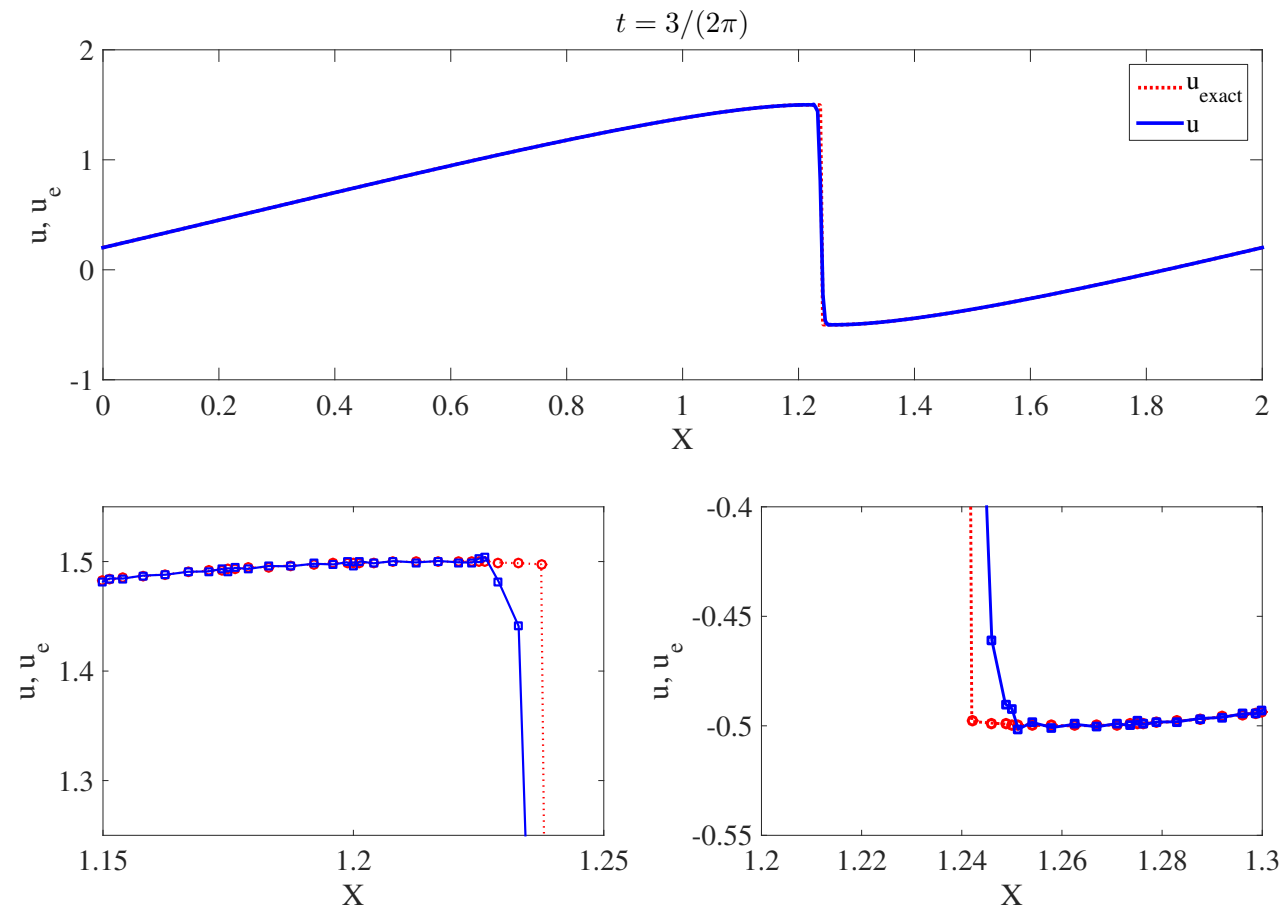

Figure 16: 1D Burgers equation at $t=3 /(2 \pi)$. Global solution and details of the discontinuity (smaller subplots). Solution computed on an 80 element grid of order 8 . The shock is well-resolved within one high-order element. The over- and under-shoots are limited, well controlled, and do not propagate from the shock across $\Omega$. The sharpness of the discontinuity is well preserved. 

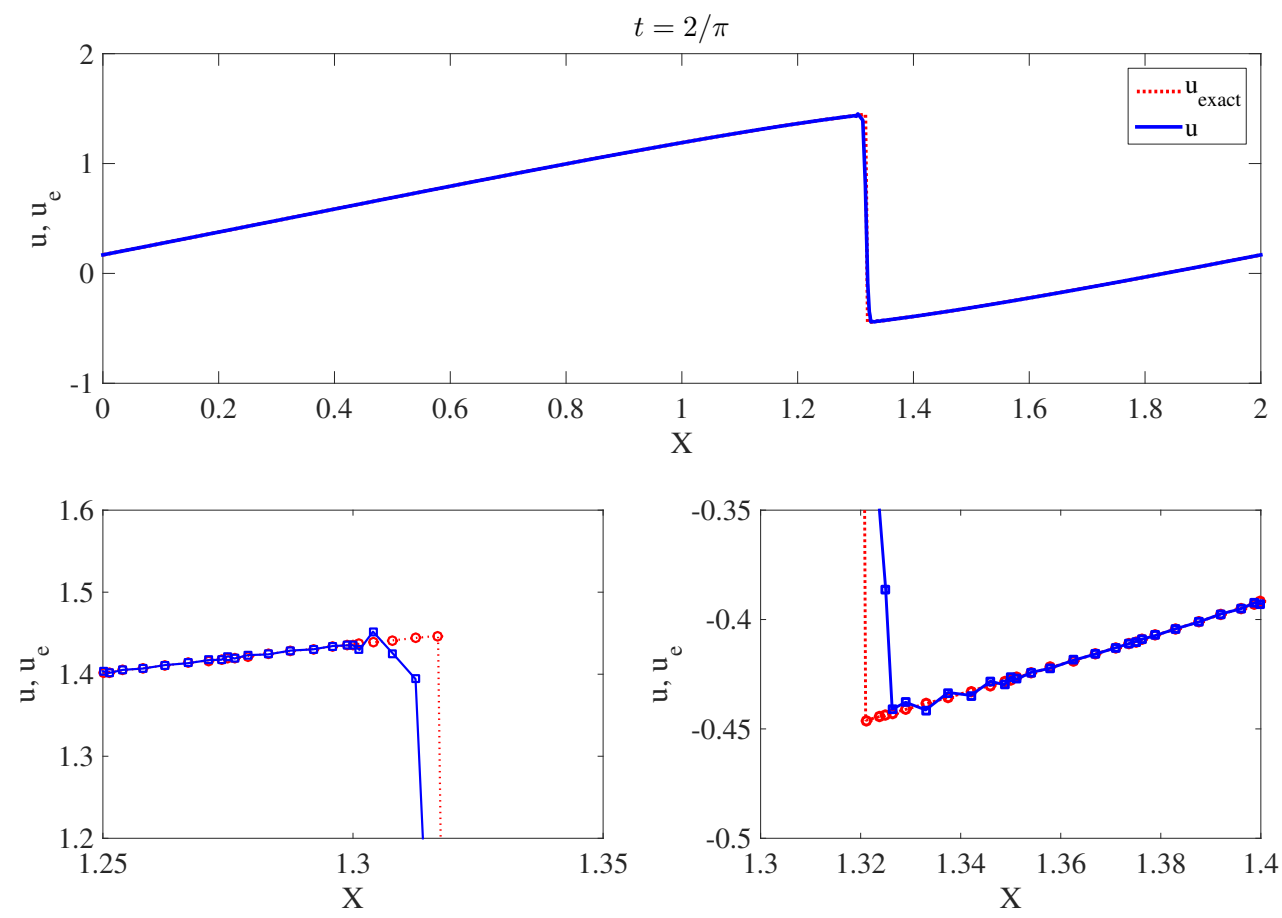

Figure 17: Like Fig. 16, but at the final time $t=2 / \pi \mathrm{s}$.
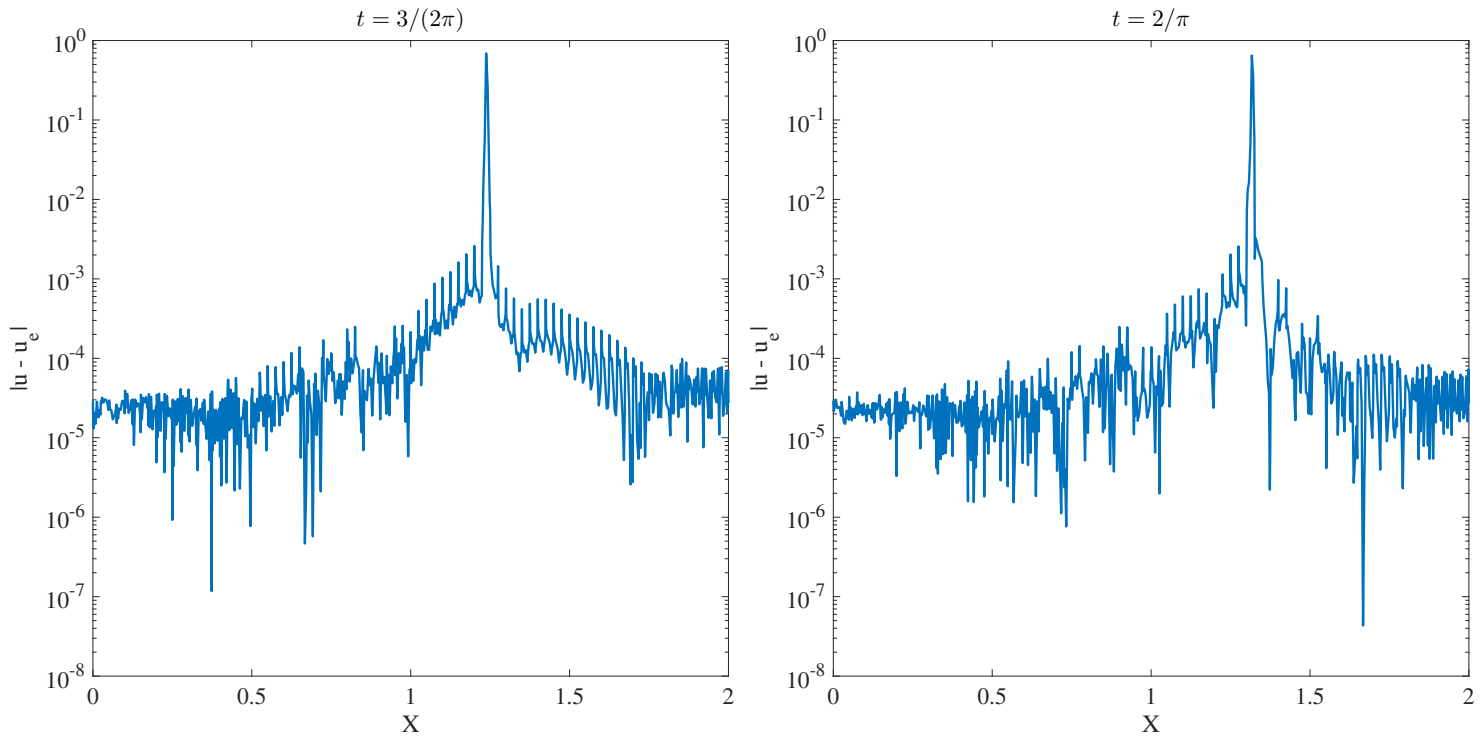

Figure 18: 1D Burgers equation. Point-wise error, $\left|u-u_{\text {exact }}\right|$, at the two time levels $t=$ $[3 /(2 \pi), 2 / \pi] \mathrm{s}$. 


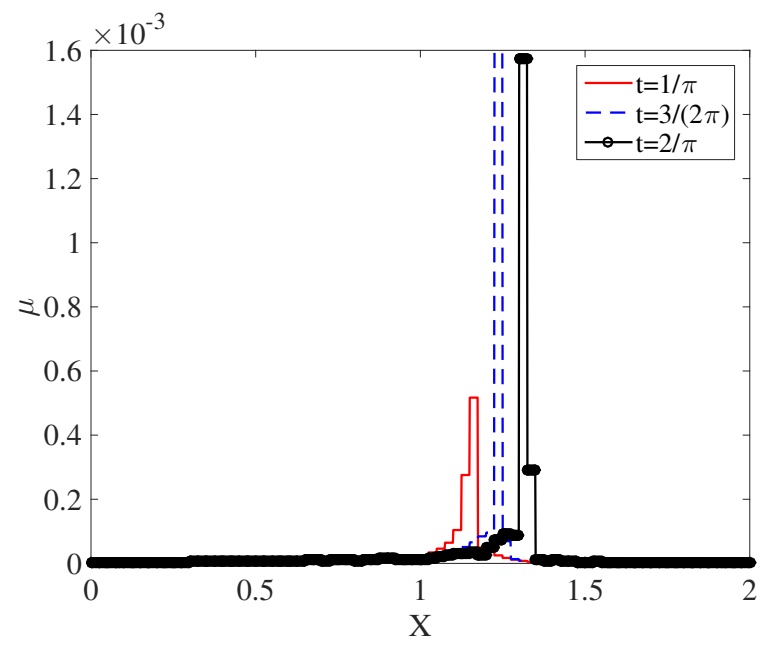

Figure 19: 1D Burgers equation. Structure of $\mu$, Eq. (15), at the three time levels $t=$ $[1 / \pi, 3 /(2 \pi), 2 / \pi] \mathrm{s}$.
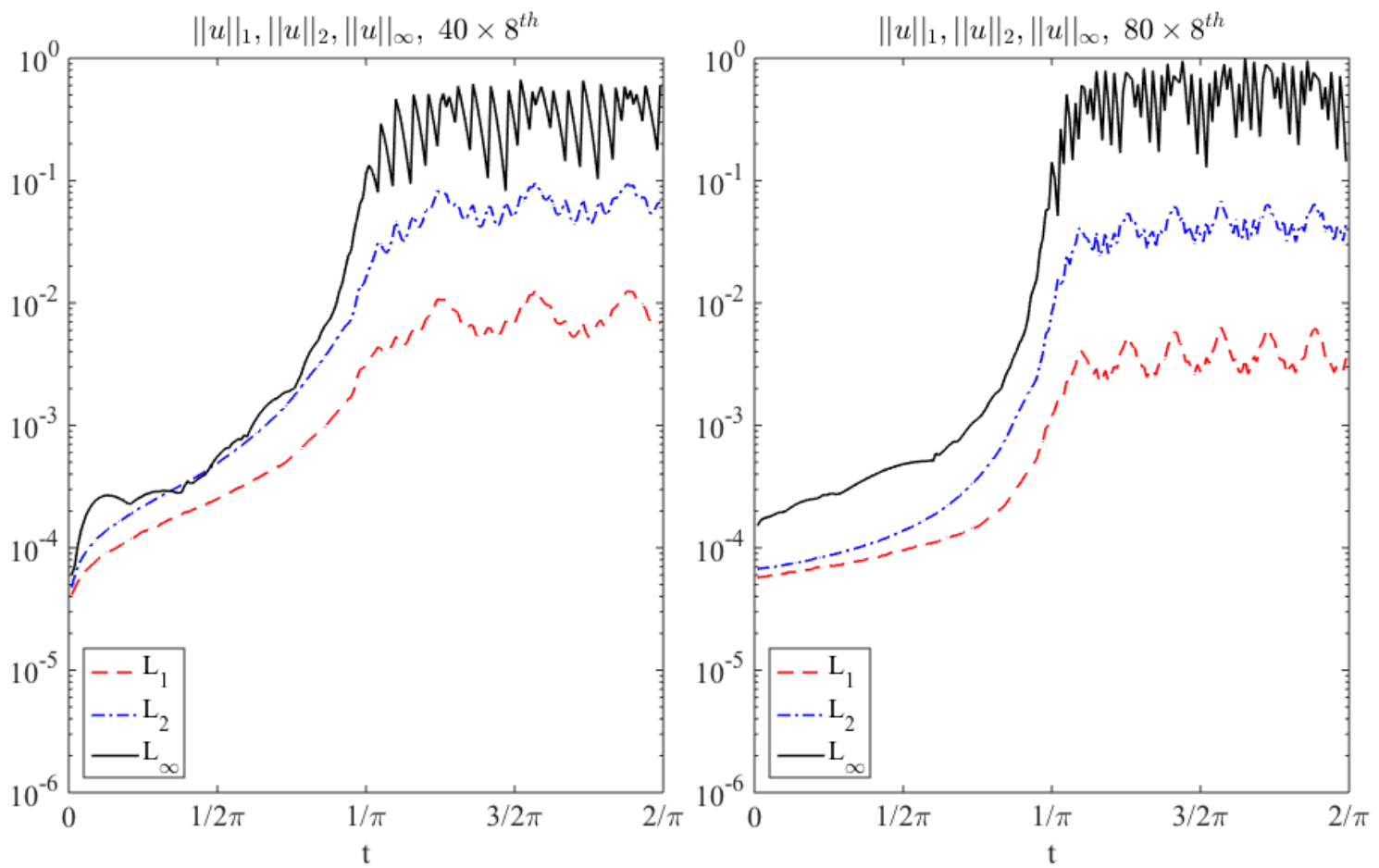

Figure 20: 1D Burgers equation. Normalized $L_{1}, L_{2}$, and $L_{\infty}$ error norms as a function of time. Left: $\Delta x=6.25 \times 10^{-3} \mathrm{~m}$. Right: $\Delta x=3.125 \times 10^{-3} \mathrm{~m}$. As expected, a large jump in the error for the infinity norm occurs as soon as the shock forms. 
when using a fourth (or higher) order hyper-diffusion. On the way towards exascale computing, fewer communications have a direct impact on the simulation speed; this factor is of fundamental importance for the design of next generation atmospheric models.

We have seen how Dyn-SGS is based on element-wise coefficients. Based on the observations of $[34 ; 3 ; 17]$, the current method (and hence the solution) would benefit if the discontinuous, elementwise viscosity could be smoothed via some proper mechanism. Taking advantage of the continuity of the solution across spectral elements, a point-wise definition of the diffusion coefficient should be sufficient. This issue will be explored in the future, together with a more thorough analysis of how Dyn-SGS performs on passive tracers and how it can be used as a turbulence model.

We have anticipated that the current dissipation is independent of the numerical method. It could be implemented in a discontinuous Galerkin, finite volume, or other environments alike. As shown in the Appendix, we have been working on its application using discontinuous Galerkin and will report more of our findings in a future paper.

\section{Acknowledgements}

The authors are thankful to the editor of this paper, Prof. Smolarkiewicz and to two anonymous reviewers whose comments helped improve the document. The discussions with and inputs by Prof. Lucas Wilcox (NPS), Dr. Matias Avila (BSC-CNS), and Dr. James F. Kelly (Exa Corp.) are also very much appreciated. The first and third authors acknowledge the support of the Office of Naval Research through program element PE-0602435N, the National Science Foundation (Division of Mathematical Sciences) through program element 121670, and the Air Force Office of Scientific Research through the Computational Mathematics program. The work of the first author was supported by the National Academies via a National Research Council fellowship.

\section{A Stabilized discontinuous Galerkin}

Dyn-SGS is independent of the numerical method. Its construction is only tied to the governing equations. To show this, we applied it to the discontinuous Galerkin solution of the rising thermal bubble problem described in [20] and solved therein using CG and DG. These results are reported in this Appendix because the application of Dyn-SGS to the discontinuous Galerkin method falls beyond the scope of this paper; nevertheless, it is meaningful to show how the viscosity described in this paper can be utilized outside the realm of finite and spectral elements. A full analysis of the performance and applicability of Dyn-SGS to DG will be specifically analyzed in a future work.

For DG, the flux form of the governing equations must be adopted, so that we re-write equations (3) in conservation form as:

$$
\begin{gathered}
\frac{\partial \bar{\rho}}{\partial t}+\frac{\bar{\rho} \widetilde{u}_{j}}{\partial x_{j}}=0 \\
\frac{\partial \bar{\rho} \widetilde{u}_{i}}{\partial t}+\frac{\bar{\rho} \widetilde{u}_{i} \widetilde{u}_{j}}{\partial x_{j}}+\frac{\partial \bar{p}}{\partial x_{i}}=-\frac{\partial \tau_{i j}^{S G S}}{\partial x_{j}}-\bar{\rho} g \delta_{i} \\
\frac{\partial \bar{\rho} \widetilde{\theta}}{\partial t}+\frac{\bar{\rho} \widetilde{\theta} \widetilde{u}_{j}}{\partial x_{j}}=-\frac{\partial Q_{j}^{S G S}}{\partial x_{j}}
\end{gathered}
$$



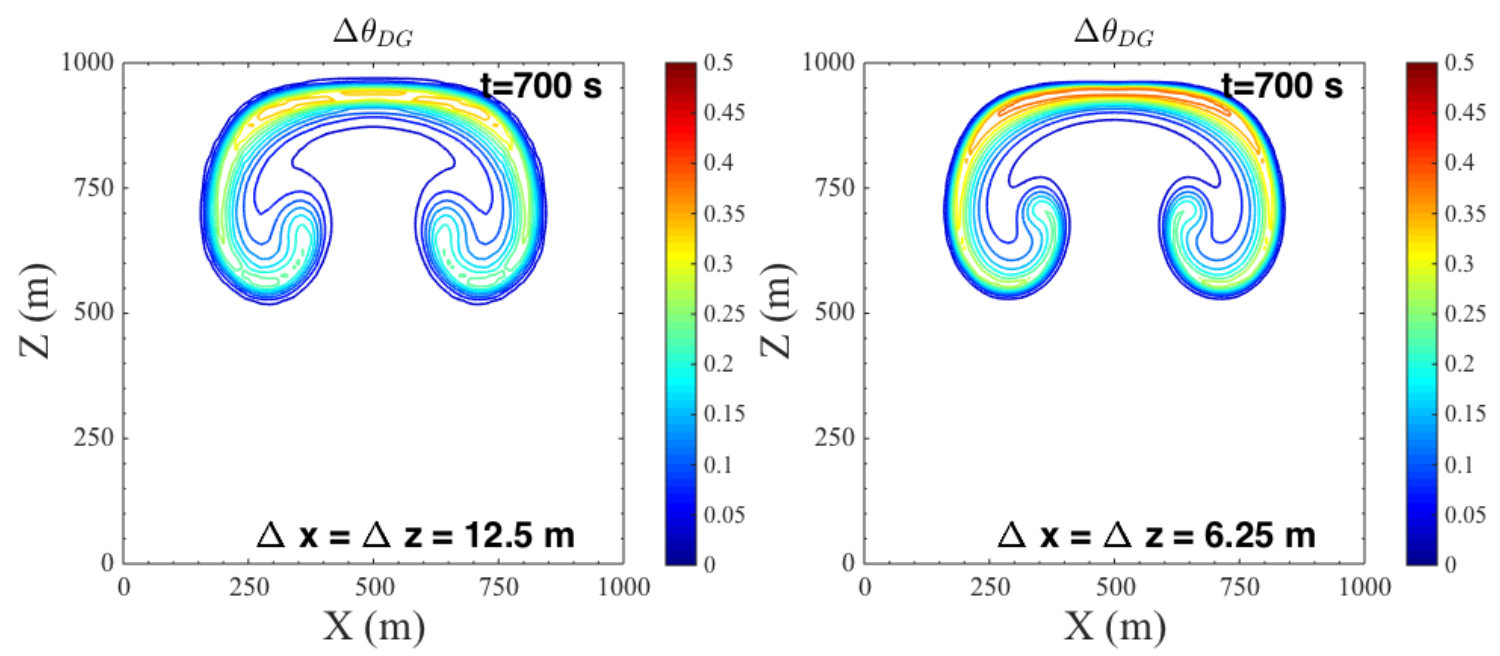

Figure 21: Rising thermal bubble in $\Omega=[1000]^{2} \mathrm{~m}^{2}$. DG solution at $\Delta \theta$ at $t=700$ for the two resolutions $\Delta x=\Delta z=12.5 \mathrm{~m}(20 \times 20$ elements of order 4$)$ and $\Delta x=\Delta z=6.25 \mathrm{~m}(40 \times 40$ elements of order 4$)$.

where $\tau_{i j}^{S G S}$ and $Q_{j}^{S G S}$ are, again, modeled as (4) and (6). The details on the DG approximation of (34) can be found in [33]. Here we simply state that the second-order viscous operators are discretized via the Local Discontinuous Galerkin (LDG) approach [11]. To build Dyn-SGS for DG, the equation residuals must account for the numerical flux that results from the DG approximation. This is necessary to remove the inherent dissipation of DG from the SGS model.

The solution is plotted in Fig. 21 for two grid resolutions: $\Delta x=\Delta z=12.5 \mathrm{~m}$, left, and $\Delta x=$ $\Delta z=6.25$, right, in the domain $\Omega=[1000]^{2} \mathrm{~m}^{2}$. In [20] the stability of the solution was achieved via a non-dissipative low pass spatial filter $[52 ; 6]$. For direct comparison, a viscous solution of the same problem is reported in [53] using a parameter-dependent element-based viscosity.

We are fully aware of the many other stabilization methods designed for discontinuous Galerkin (DG) (see, e.g., [44; 34]); however, the fact that Dyn-SGS is based on the physical Navier-Stokes stress operators and seems feasible for large eddy simulations, merits consideration for proposing another stabilizing scheme for DG.

\section{References}

[1] R. Abgrall. Toward the ultimate conservative scheme: following the quest. J. Comput. Phys., 167:277-315, 2001.

[2] N. Ahmad and J. Lindeman. Euler solutions using flux-based wave decomposition. Int. J. Numer. Meth. Fluids, 54:47-72, 2007.

[3] G. E. Barter and D. L. Darmofal. Shock capturing with PDE-based artificial viscosity for DGFEM: Part I. Formulation. J. Comput. Phys., 229:1810-1827, 2010. 
[4] H. Bateman. Some recent researches on the motion of fluids. Mon. Wea. Rev., 43:163-170, 1915.

[5] P. Benoit and S. Chi-Wang. On positivity preserving finite volume schemes for Euler equations. Numer. Math., 73(1):119-130, 1996.

[6] J. P. Boyd. The erfc-log filter and the asymptotics of the Euler and Vandeven sequence accelerations. In A.V. Ilin, L.R. Scott (Eds.), Proceedings of the Third International Conference on Spectral and High Order Methods, Houston Journal of Mathematics, pages 267-276, 1996.

[7] A N Brooks and T J R Hughes. Streamline upwind/Petrov-Galerkin formulations for convective dominated flows with particular emphasis on the incompressible navier-stokes equations. Comput. Methods Appl. Mech. Eng., 32:199-259, 1982.

[8] J. M. Burgers. A mathematical model illustrating the theory of turbulence. Adv. Appl. Mech., $1: 171-199,1948$.

[9] V. M. Canuto and Y. Cheng. Determination of the Smagorinsky-Lilly constant $C_{S}$. Physics of Fluids, 9:1368-1378, 1997.

[10] R. Carpenter, K. Droegemeier, P. Woodward, and C. Hane. Application of the piecewise parabolic method (PPM) to meteorological modeling. Mon. Wea. Rev., 118:586-612, 1990.

[11] B. Cockburn and C W. Shu. The local discontinuous Galerkin method for time-dependent convection-diffusion systems. SIAM J. Numer. Anal., 35:2440-2463, 1998.

[12] R. Courant, K. Friedrichs, and H. Lewy. On the partial difference equations of mathematical physics. IBM Journal: translation from the original paper in Mathematische Annale, 100,3224, 100:215-234, 1928.

[13] J. W. Deardorff. A numerical study of three-dimensional turbulent channel flow at large Reynolds numbers. J. Fluid Mech., 41:452-480, 1970.

[14] M. O. Deville, P. F. Fischer, and E. H. Mund. High-order methods for incompressible fluid flow. Cambridge University Press, 2002.

[15] A. Ern and J. L. Guermond. Weighting the edge stabilization. SIAM J. Numer. Anal., 51:1655$1677,2013$.

[16] A. Favre. Turbulence: space-time statistical properties and behavior in supersonic flows. Phys. Fluids, 26:2851-2863, 1983.

[17] M. Feistauer and Kučera. On a robust discontinuous Galerkin technique for the solution of compressible flow. J. Comput. Phys., 224:208-221, 2007.

[18] E. Garnier, N. Adams, and P. Sagaut. Large Eddy Simulation for compressible flows. Springer, 2009.

[19] F X. Giraldo, J F.. Kelly, and E. Constantinescu. Implicit-explicit formulations of a threedimensional Nonhydrostatic Unified Model of the Atmosphere (NUMA). SIAM J. Sci. Comput., 35:1162-1194, 2013. 
[20] F. X. Giraldo and M. Restelli. A study of spectral element and discontinuous Galerkin methods for the Navier-Stokes equations in nonhydrostatic mesoscale atmospheric modeling: Equation sets and test cases. J. Comput. Phys., 227:3849-3877, 2008.

[21] O. Guba, M. A. Taylor, P. A. Ullrich, J. R. Overfelt, and M. N. Levy. The spectral element method on variable resolution grids: evaluating grid sensitivity and resolution-aware numerical viscosity. Geosci. Model Dev. Discuss., 7:4081-4117, 2014.

[22] J L. Guermond and R. Pasquetti. Entropy-based nonlinear viscosity for Fourier approximations of conservation laws. C. R. Acad. Sci., Ser. I, 346:801-806, 2008.

[23] J L. Guermond, R. Pasquetti, and B. Popov. Entropy viscosity method for nonlinear conservation laws. J. Comput. Phys., 230:4248-4267, 2011.

[24] J L. Guermond and B. Popov. Viscous regularization of the Euler equations and entropy principles. SIAM J. Appl. Math., 74(2):284-305, 2014.

[25] J. Holton. An introduction to dynamic meteorology. Elsevier Academic Press: Internation Geophysics Series: Vol. 88, 4th edition, 2004.

[26] J. Houghton. Physics of atmospheres. Cambridge University Press, 3rd Ed., 2002.

[27] G. Houzeaux and J. Principe. A variational subgrid scale model for transient incompressible flows. International Journal of Computational Fluid Dynamics, 22:135-152, 2008.

[28] T. J. R. Hughes. Multiscale phenomena: Green's functions, the Dirichlet-to-Neumann formulation, subgrid scale models, bubbles and the origins of stabilized methods. Comput. Methods Appl. Mech. and Engrg., 127:387-401, 1995.

[29] T J R Hughes, L P Franca, and G M Hulbert. A new finite element formulation for computational fluid dynamics: III. the Galerkin/least-squares method for advection-diffusive equations. Comput. Methods Appl. Mech. Eng., 73:329-336, 1989.

[30] C. Jablonowski and D. L. Williamson. A baroclinic instability test case for atmospheric model dynamical cores. Q. J. R. Meteorol. Soc., 132:2943-2975, 2006.

[31] C. Jablonowski and D. L. Williamson. The pros and cons of diffusion, filters and fixers in atmospheric general circulation models. In P H. Lauritzen, C. Jablonowski, M A. Taylor, and R D. Nair, editors, Numerical Techniques for Global Atmospheric Models, volume 80 of Lecture notes in computational science and engineering, pages 381-482. Springer, 2011.

[32] G. Karniadakis and S. Sherwin. Spectral/hp element methods for CFD. Oxford University Press, 1999.

[33] J. F. Kelly and F. X. Giraldo. Continuous and discontinuous Galerkin methods for a scalable three-dimensional nonhydrostatic atmospheric model: limited-area mode. J. Comput. Phys., 231:7988-8008, 2012.

[34] A. Klöckner, T. Warburton, and J. S. Hesthaven. Viscous shock capturing in a time-explicit discontinuous Galerkin method. Math. Model. Nat. Phenom., 10:1-27, 2011. 
[35] A. Leonard. Energy cascade in large eddy simulations of turbulent fluid flows. Advan. Geophys., 18:237-248, 1974.

[36] M. Lesieur and O. Metais. New trends in large-eddy simulations of turbulence. Ann. Rev. Fuild Mech., 28:45-82, 1996.

[37] D. K. Lilly. On the numerical simulation of buoyant convection. Tellus, 14:148-172, 1962.

[38] G. I. Marchuk. Numerical Methods in Weather Prediction. Academic Press, 1974.

[39] S. Marras and F. X. Giraldo. Towards an improved high-order transport scheme for tracers. Technical Report 062015, Naval Postgraduate School, Dept. of Applied Mathematics. Monterey, CA, U.S.A., 2015.

[40] S. Marras, J.F. Kelly, M. Moragues, A. Müller, M.A. Kopera, M. Vázquez, F.X. Giraldo, G. Houzeaux, and O. Jorba. A review of element-based Galerkin methods for Numerical Weather Prediction. finite elements, spectral elements, and discontinuous Galerkin. Arch. Comput. Methods Engrg. (in press, DOI: 10.1007/s11831-015-9152-1), pages 1-50, 2015.

[41] S. Marras, M. Moragues, M. Vázquez, O. Jorba, and G. Houzeaux. A variational multiscale stabilized finite element method for the solution of the Euler equations of nonhydrostatic stratified flows. J. Comput. Phys., 236:380-407, 2013.

[42] M. Nazarov. Convergence of a residual based artificial viscosity finite element method. Comput. Math. Appl., 65(4):616-626, 2013.

[43] M. Nazarov and J. Hoffman. Residual-based artificial viscosity for simulation of turbulent compressible flow using adaptive finite element methods. Int. J. Numer. Methods Fluids, 71:339-357, 2013.

[44] P. Persson and J. Peraire. Sub-cell shock capturing for discontinuous Galerkin methods. In Proc. of the 44th AIAA Aerospace Sciences Meeting and Exhibit, volume 112, 2006.

[45] F. Rispoli and R. Saavedra. A stabilized finite element method based on sgs models for compressible flows. Comp. Meth. Appl. Mech. Engrg., 196:652-664, 2006.

[46] A. Scotti, C. Meneveau, and D. K. Lilly. Generalized Smagorinsky model for anisotropic grids. Phys. Fluids, 5:2306-2308, 1993.

[47] J. Smagorinsky. General circulation experiments with the primitive equations: I. the basic experiement. Mon. Wea. Rev., 91:99-164, 1963.

[48] J. Straka, R. Wilhelmson, L. Wicker, J. Anderson, and K. Droegemeier. Numerical solution of a nonlinear density current: a benchmark solution and comparisons. Int. J. Num. Meth. in Fluids, 17:1-22, 1993.

[49] E. Tadmor. A minimum entropy principle in the gas dynamics equations. Appl. Numer. Math., $2(3-5): 211-219,1986$.

[50] E. Tadmor. Convergence of spectral methods for nonlinear conservation laws. SIAM J. Numer. Anal., 26:30-44, 1989. 
[51] P A. Ullrich and C. Jablonowski. Operator-split Runge-Kutta-Rosenbrock methods for nonhydrostatic atmospheric models. Mon. Wea. Rev., 140:1257-1284, 2012.

[52] H. Vandeven. Family of spectral filters for discontinuous problems. J. Sci. Comp., 6:159-192, 1991.

[53] M. Yu, F X. Giraldo, M. Peng, and Z J. Wang. Localized artificial viscosity stabilization of discontinuous Galerki n mehods for nonhydrostatic mesoscale atmospheric modeling. Technical report, Kansas University, 2014. 